\title{
Symmetric invariants and cohomology of groups
}

\author{
Alejandro Adem, ${ }^{1, \star}$ John Maginnis, ${ }^{2, \star}$ and R. James Milgram ${ }^{3, \star}$ \\ 1 School of Mathematics, Institute for Advanced Study, Princeton NJ 08540, USA, and Mathematics \\ Department, University of Wisconsin-Madison, Madison, WI 53706, USA \\ 2 Mathematics Department, University of Michigan, Ann Arbor, MI 48109, USA \\ ${ }^{3}$ Mathematics Department, Stanford University, Stanford, CA 94305, USA
}

\section{Introduction}

Some time ago Nakaoka $[\mathrm{N}]$ determined the additive structure of $H^{*}\left(\Sigma_{n}, \mathrm{~F}_{2}\right)$. Around the same time, the ring structure of $H^{*}\left(\Sigma_{4}, \mathrm{~F}_{2}\right)$ was calculated and it was assumed that the same type of simple multiplicative relations would appear for $n>4$. Since then, most work has concentrated on the embedding $\Sigma_{n} \hookrightarrow \Sigma_{\infty}$, using the polynomial ring structure of $H^{*}\left(\Sigma_{\infty}, \mathrm{F}_{2}\right)$. In particular, most of the recent work in $[\mathrm{H}]$ was explicitly or implicitly described in $[\mathbf{M}-\mathbf{M}]$ more than a decade ago, and provides no new information on the multiplicative structure of the mod 2 cohomology of finite symmetric groups.

In this paper we use invariant theory to detect unexpected multiplicative relations in $H^{*}\left(\Sigma_{n}, \mathbf{F}_{2}\right)$. The complicated nature of the numerical evidence which we present explains the absence of complete calculations for $n \geqq 16$. The rings of invariants which we study are at the core of any computation of $H^{*}\left(\Sigma_{n}, F_{2}\right)$. They build up sucessively, yielding relations rich in symmetry but of a highly convoluted type.

The following problem lies at the heart of all this. Let $\boldsymbol{P}_{n}$ be a polynomial ring over $F_{2}$ on $n$ variables, and let $P_{n, k}=\bigotimes_{1}^{k} P_{n}$. Then $\Sigma_{k}$ acts by permuting blocks of generators.

Problem. Determine $\left(P_{n, k}\right)^{\Sigma_{k}}$.

The fundamental theorem of Galois theory states that for $n=1$, any $k$, this is a polynomial ring. Our results show that this breaks down even for $n=2$ and that things get quite complicated quickly. For $n=2$ and $k=2$ we exhibit a quadratic relation, which yields the first relation of this sort in the cohomology of the symmetric groups. Concretely, we have

Theorem 3.2.

$$
H^{*}\left(\Sigma_{8}\right) \cong P\left[\sigma_{1}, \sigma_{2}, \sigma_{3}, c_{3}, \sigma_{4}, d_{6}, d_{7}\right]\left(x_{5}\right) /\langle R\rangle
$$

where $\operatorname{deg} \sigma_{i}=i, \operatorname{deg} c_{3}=3, \operatorname{deg} d_{i}=i, \operatorname{deg} x_{5}=5$, and $R$ is the following set of

\footnotetext{
* Partially supported by NSF grants (all three authors) and the S.F.B. 170 (the third author)
} 
relations:

$$
\begin{aligned}
& d_{6} \sigma_{1}=d_{6} \sigma_{3}=0 \\
& d_{7} \sigma_{1}=d_{7} \sigma_{2}=d_{7} \sigma_{3}=d_{7} c_{3}=d_{7} x_{5}=0 \\
& x_{5} \sigma_{3}+c_{3} \sigma_{4} \sigma_{1}=0 \\
& c_{3}\left(\sigma_{3}+\sigma_{1} \sigma_{2}\right)+\sigma_{1} x_{5}=0 \\
& x_{5}^{2}+x_{5} \sigma_{2} c_{3}+d_{6} \sigma_{2}^{2}+\sigma_{4} c_{3}^{2}=0 .
\end{aligned}
$$

This is a formal description as a ring. However, the explicit generators are described in Sect. 1 as duals to certain monomials in the Dyer-Lashof algebra $H_{*}\left(\Sigma_{\infty}, \mathbf{F}_{2}\right) \cong H_{*}\left(Q\left(S^{0}\right)_{0}, \mathbf{F}_{2}\right)$, where $Q\left(S^{0}\right)$ denotes the infinite loop space $\Omega^{\infty} \Sigma^{\infty}\left(S^{0}\right)$. An alternative algebraic description is also supplied. We compute $\left(P_{2,3}\right)^{\Sigma_{3}}$ similarly, and use this to obtain $H^{*}\left(\Sigma_{12}, \mathrm{~F}_{2}\right)$ :

\section{Theorem 3.5 .}

$$
H^{*}\left(\Sigma_{12}\right) \cong P\left[\sigma_{1}, \sigma_{2}, \sigma_{3}, c_{3}, \sigma_{4}, \sigma_{5}, \sigma_{6}, d_{6}, d_{7}, d_{9}\right]\left(x_{5}, x_{7}, x_{8}\right) /\langle R\rangle
$$

where $\operatorname{deg} \sigma_{i}=i, \operatorname{deg} c_{3}=3, \operatorname{deg} d_{i}=i, \operatorname{deg} x_{i}=i$ and $R$ is the set of relations

$$
\begin{aligned}
& x_{7}^{2}+\sigma_{4} x_{5}^{2}+\sigma_{2} \sigma_{4} x_{8}+\left(\sigma_{6} c_{3}+\sigma_{2} \sigma_{4} c_{3}\right) x_{5}+\sigma_{4}^{2}+c_{3}^{2}+\sigma_{2}^{2} \sigma_{4} d_{6}+\sigma_{6} \sigma_{2} d_{6}, \\
& x_{8}^{2}+d_{6} x_{5}^{2}+c_{3} d_{6} x_{7}+\left(d_{9} \sigma_{2}+c_{3} d_{6} \sigma_{2}\right) x_{5}+d_{6}^{2} \sigma_{2}^{2}+c_{3}^{2} \sigma_{4} d_{6}+d_{9} c_{3} \sigma_{2}, \\
& x_{5} x_{7}+\sigma_{2} x_{5}^{2}+\left[\sigma_{2}^{2}+\sigma_{4}\right] x_{8}+\sigma_{2}^{2} c_{3} x_{5}+\sigma_{2}^{3} d_{6}+\sigma_{2} d_{6} \sigma_{4}+\sigma_{6} c_{3}^{2}+\sigma_{6} d_{6}+\sigma_{2} \sigma_{4} c_{3}^{2}, \\
& x_{5} x_{8}+c_{3} x_{5}^{2}+\left[c_{3}^{2}+d_{6}\right] x_{7}+c_{3}^{2} \sigma_{2} x_{5}+c_{3}^{3} \sigma_{4}+c_{3} \sigma_{4} d_{6}+d_{9} \sigma_{2}^{2}+d_{9} \sigma_{4}+c_{3} d_{6} \sigma_{2}^{2}, \\
& x_{5}^{3}+c_{3} \sigma_{2} x_{5}^{2}+x_{7} x_{8}+c_{3} \sigma_{2}^{2} x_{8}+\sigma_{2} c_{3}^{2} x_{7}+\left(\sigma_{2}^{2} d_{6}+\sigma_{4} c_{3}^{2}\right) x_{5}+\sigma_{2}^{3} d_{9}+c_{3}^{3} \sigma_{6}+\sigma_{6} d_{9}, \\
& d_{9} \sigma_{1}, d_{9} \sigma_{3}, d_{9} \sigma_{5}, \\
& d_{7} \sigma_{3}, d_{7} x_{5}, d_{7} \sigma_{1} c_{3}, d_{7}\left(x_{7}+\sigma_{4} c_{3}\right), d_{7}\left(\sigma_{5}+\sigma_{4} \sigma_{1}\right), d_{7}\left(\sigma_{6}+\sigma_{4} \sigma_{2}\right), d_{7}\left(x_{8}+d_{6} \sigma_{2}\right), \\
& d_{7}\left(d_{9}+d_{6} c_{3}\right), \\
& x_{7} \sigma_{1}+x_{5}\left(\sigma_{1} \sigma_{2}+\sigma_{3}\right)+c_{3}\left(\sigma_{2} \sigma_{3}+\sigma_{2}^{2} \sigma_{1}+\sigma_{1} \sigma_{4}+\sigma_{5}\right), \\
& x_{7} \sigma_{3}+x_{5}\left(\sigma_{5}+\sigma_{1} \sigma_{4}\right)+c_{3}\left(\sigma_{1} \sigma_{6}+\sigma_{3} \sigma_{4}+\sigma_{1} \sigma_{2} \sigma_{4}\right), \\
& x_{7} \sigma_{5}+x_{5} \sigma_{1} \sigma_{6}+c_{3}\left(\sigma_{3} \sigma_{6}+\sigma_{1} \sigma_{2} \sigma_{6}\right), \\
& x_{8} \sigma_{1}+d_{6}\left(\sigma_{3}+\sigma_{1} \sigma_{2}\right), \\
& x_{8} \sigma_{3}+d_{6}\left(\sigma_{5}+\sigma_{1} \sigma_{4}\right), \\
& x_{8} \sigma_{5}+d_{6} \sigma_{1} \sigma_{6} . \quad \square
\end{aligned}
$$

For completeness we also indicate $H^{*}\left(\Sigma_{6}\right), H^{*}\left(\Sigma_{10}\right)$ in 3.1 and 3.4.

These rings of invariants represent the image of the restriction map in the cohomology of certain elementary abelian subgroups, which detect the multiplicative relations. For $n=2$ and $k=4,5,6$ we have numerical data which indicates a highly non-trivial ring structure for $H^{*}\left(\Sigma_{16}, \mathrm{~F}_{2}\right)$ and beyond. We hope to recognize general patterns which will enable us to extend our computations. It is well known that the mod 2 cohomology of the symmetric groups is detected on elementary abelian subgroups, and the additive structure can be determined from this. Generators appearing at a finite stage will exist stably, but the precise multiplicative relations among them change at each level, as the lattice of detecting subgroups becomes larger and more complicated. 
Let $G$ be a finite group, then there is a canonical embedding $G \subset S_{|G|}$ induced by the regular representation. If $H \subseteq G$, we have a commutative diagram

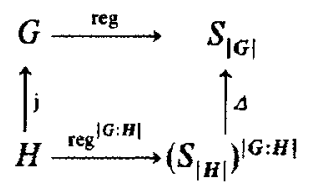

Indeed, we can write $G=H g_{1} \cup H g_{2} \cup \cdots \cup H g_{[G: H]}$, where the $g_{i}$ are representatives for the right cosets of $H$ in $G$. Then, for $g \in H$, acting from the left on $G$, we clearly have the asserted diagram. These maps give us canonical characteristic classes for group cohomology. For example, if $G=(\mathrm{Z} / 2)^{n}$, then im reg* $=H^{*}\left((\mathbf{Z} / 2)^{n}, \mathbf{F}_{2}\right)^{G L_{n}\left(F_{2}\right)}$ the Dickson algebra. Note that $N_{S_{|G|}}(\operatorname{reg}(G))=$ Aut $G$, in particular im reg* $^{*} \subseteq H^{*}(G)^{\text {Out(G) }}$. This may partly explain why the Dickson algebra plays such an important role. Also note that from the above it follows that im reg* is a large portion of the non-nilpotent part of $H^{*}\left(G, F_{2}\right)$.

On the other hand, given any embedding $G \stackrel{\dot{C}}{\hookrightarrow} \Sigma_{n}$, we know from a result due to Evens [E] that under the induced map $i^{*}: H^{*}\left(\Sigma_{n}\right) \rightarrow H^{*}(G)$, the cohomology of $G$ is a finitely generated $H^{*}\left(\Sigma_{n}\right)$-module. Hence the cohomology rings of the finite symmetric groups carry universal relations which will affect the cohomology of their subgroups. The situation is a lot more delicate than that induced by the embeddings $G \hookrightarrow S_{\infty}$ or $G \leftrightharpoons U(n)$, as the cohomology of these larger groups are free abelian algebras: tensor products of polynomial algebras on even dimensional generators and exterior algebras on odd ones at odd primes, while for $\mathbf{Z} / 2$ coefficients $H^{*}\left(\Sigma_{\infty}\right)$ is a polynomial ring on generators dual to the generators of the Dyer-Lashof algebra [M-M]. We are currently analyzing the general pattern for all symmetric groups, which, if understood, would provide insight into the cohomological behaviour of arbitrary finite groups.

Calculating the cohomology of a simple group is especially hard, as one cannot make use of the Lyndon-Hochschild-Serre spectral sequence. In Sect. 4 we use our results on the symmetric groups to give an explicit method for determining the additive structure of $H^{*}\left(A_{n}, \mathrm{~F}_{2}\right)$, any $n$. We also describe the mod 2 cohomology rings of $A_{8}, A_{10}$, and $A_{12}$, which have been of interest to algebraists.

\section{Corollary 4.5.}

$$
H^{*}\left(A_{8}\right) \cong P\left[\sigma_{2}, c_{3}, \sigma_{3}, \sigma_{4}, d_{6}, e_{6}, d_{7}, e_{7}\right]\left(x_{5}\right) /\langle R\rangle
$$

where $\operatorname{deg} \sigma_{i}=i, \operatorname{deg} c_{3}=3, \operatorname{deg} d_{i}=i, \operatorname{deg} e_{i}=i, \operatorname{deg} x_{i}=5$, and $R$ is the following set of relations:

$$
\begin{aligned}
& d_{6} \sigma_{3}=0, d_{6} d_{7}+d_{6} e_{7}+e_{6} e_{7}=0, d_{6}^{2}+d_{6} e_{6}+e_{6}^{2}=0, \\
& d_{7} \sigma_{2}=d_{7} \sigma_{3}=d_{7} c_{3}=d_{7} x_{5}=0, d_{6} d_{7}+d_{7} e_{6}+e_{6} e_{7}=0, d_{7}^{2}+d_{7} e_{7}+e_{7}^{2}=0, \\
& e_{6} \sigma_{3}=0 \\
& e_{7} \sigma_{2}=e_{7} \sigma_{3}=e_{7} c_{3}=e_{7} x_{5}=0 \\
& x_{5} \sigma_{3}=0, c_{3} \sigma_{3}=0 \\
& x_{5}^{2}+x_{5} \sigma_{2} c_{3}+\left(d_{6}+e_{6}\right) \sigma_{2}^{2}+\sigma_{4} c_{3}^{2}=0 .
\end{aligned}
$$


Tezuka and Yagita [T-Y] caiculated the mod 2 cohomology of $G L_{4}\left(F_{2}\right)$. Using the classical isomorphism $A_{8} \cong G L_{4}\left(F_{2}\right)$ we obtain a much cleaner and precise description, correcting some imprecisions. We obtain our result using $H^{*}\left(\Sigma_{8}, \mathbf{F}_{2}\right)$ and a short exact sequence in cohomology which exists in the index two situation. We use the same method to determine the cohomology of $A_{10}, A_{12}$.

We would like to point out that our calculations were motivated by specific questions in topology. We required precise understanding of $H^{*}\left(\Sigma_{n}, \mathrm{~F}_{2}\right)$ as rings and modules over the Steenrod algebra (which we obtain in all the examples mentioned previously) in order to analyze certain double covers $\tilde{\Sigma}_{n}$ which serve as finite models for a classifying space which, among other things, serves to detect the Kirby-Siebenmann characteristic class of 4-dimensional manifolds (see [M1] for more on this). We hope to elaborate on these applications in a separate paper.

It is also interesting to note that the smaller alternating and symmetric groups are intimately involved in the structure of many sporadic and Lie type simple groups. For example, there is a subgroup of the form $(Z / 2)^{4} \times{ }_{T} A_{6}$ in the Mathieu group $M_{22}$, with odd index, and the stability conditions to determine the exact image of the restriction map in mod 2 cohomology can be described explicitly (see [AMM], Sect. 3). Also (Z/2) ${ }^{4} \times{ }_{T} A_{7}$ is a subgroup of odd index in $M_{23}$, so a similar analysis can be made. The double cover of $A_{8}, \tilde{A}_{8}$ is a subgroup of $\mathrm{McL}$, and shares the same 2-Sylow subgroup with $M_{22}$. Finally we note that $\tilde{A}_{10} \subset L y$, again with odd index. It seems likely that combining the approach described in [AMM] with the results in this paper, one can obtain substantial information about the cohomology of the groups mentioned above.

The organization of the paper is as follows: in Sect. 1 we briefly describe the determination of the additive structure of $H^{*}\left(\Sigma_{n}, \mathbf{F}_{2}\right)$; in Sect. 2 we explain the invariant theory necessary to detect multiplicative relations; in Sect. 3 we calculate $H^{*}\left(\Sigma_{n}, \mathrm{~F}_{2}\right), n=6,8,10,12$ as rings and modules over $A(2)$; and in Sect. 4 we apply these results to find the cohomology of the alternating groups.

We would like to thank Ronnie Lee for stimulating our interest in the cohomology of simple groups, and Stewart Priddy for helpful comments. Throughout this paper $\mathbf{F}_{2}$-coefficients will be used, so we drop them from our notation.

\section{The homology of $\Sigma_{n}$}

In this section we will briefly outline how $H_{*}\left(\Sigma_{n}\right)$ can be computed using infinite loop spaces. The original calculation of $H_{*}\left(\Sigma_{\infty}\right)$ is due to Nakaoka [N], but we choose to follow the approach due to Milgram [M], Barratt-Priddy [B-P] and Quillen [Q] which yields the homology of the symmetric groups as a corollary of fundamental results in homotopy theory. The reason is that this point of view is more conceptual and will serve as background for a sequel, where we discuss topological consequences of our calculations.

If $X$ is a space with basepoint, the natural inclusions $\Omega^{n} S^{n}(X) \rightarrow \Omega^{n+1} S^{n+1}(X)$ on passing to the limit define the space $Q(X)=\lim _{n \rightarrow \infty} \Omega^{n} S^{n}(X)$. $Q(X)$ depends functorially on $X$ and $\pi_{i}(Q(X))=\pi_{i}^{s}(X)$, the $i$-th stable homotopy group of $X$. Let 
$C\left(S^{\circ}\right)=\coprod_{n \geqq 0} B \Sigma_{n}$, the disjoint union, with $B \Sigma_{0}$ as base point $*$. The usual inclusions $\Sigma_{n} \times \Sigma_{m} \rightarrow \Sigma_{n+m}$ induce maps $B \Sigma_{n} \times B \Sigma_{m} \rightarrow B \Sigma_{n+m}$ which together with * make $C\left(S^{\circ}\right)$ into an associative $H$-space with unit (in fact, a unitary monoid). Now $Q\left(S^{\circ}\right)$ has a "loop sum product", which is given as the limit of the $\Omega^{n-1}(*): \Omega^{n} S^{n} \times \Omega^{n} S^{n} \rightarrow$ $\Omega^{n} S^{n}$ where $*: \Omega S^{n} \times \Omega S^{n} \rightarrow \Omega S^{n}$ is the loop sum. We have the following fundamental result

Theorem 1.1 (Dyer-Lashof). $Q\left(S^{\circ}\right)$ is the group completion of $C\left(S^{\circ}\right)$ and hence

$$
H_{*}\left(Q\left(S^{\circ}\right)\right) \cong\left(\lim _{n \rightarrow \infty} H_{*}\left(\Sigma_{n}\right)\right) \otimes \mathbf{F}_{2}[\mathbf{Z}] .
$$

Although the language was different, this result first appeared in the original 1960 preprint of [D-L]. Later it was independently rediscovered by Barratt-Priddy and Quillen. This is an isomorphism compatible with the two ring structures induced by the products on either side. Furthermore, the proof of this result shows how to distill $H_{*}\left(\Sigma_{n}\right)$ from the above. First we describe $H_{*}\left(Q\left(S^{\circ}\right)\right)$ under loop sum.

Theorem 1.2. Under the operation * induced by loop sum,

$$
H_{*}\left(Q\left(S^{\circ}\right)\right) \cong P\left[Q_{I}\right] \otimes \mathbf{Z} / 2[\mathrm{Z}]
$$

where $I=\left(i_{1}, \ldots, i_{n}\right)$ is a sequence of integers with $0<i_{1} \leqq i_{2} \leqq \cdots \leqq i_{n}$ and $\operatorname{deg} Q_{1}=i_{1}+2 i_{2}+\cdots+2^{n-1} i_{n}$.

For readers acquainted with Dyer-Lashof operations, we point out that $Q_{I}=Q_{i_{1}} Q_{i_{2}} \cdots Q_{i_{n}}[1]$. Note also that using the loop product we can discard the classes with $i_{1}=0$, as $Q_{\left(0, i_{2}, \ldots, i_{n}\right)}=Q_{\left(i_{2}, \ldots, i_{n}\right)} * Q_{\left(i_{2}, \ldots, i_{n}\right)}$ (a loop square). We refer to $[\mathrm{M}-\mathrm{M}]$, pg. 137 for details. Note that 1.2 implies that $H^{*}\left(\Sigma_{\infty}\right) \cong P\left[Q_{I}\right]$, a polynomial algebra on an infinite set of generators; the same is true of the cohomology ring $H^{*}\left(\Sigma_{\infty}\right)$ : The map induced in homology by the inclusion $\Sigma_{m} \hookrightarrow \Sigma_{\infty}$ is injective, and its image ie explicit, hence allowing us to compute $H^{*}\left(\Sigma_{m}\right)$. Partition $m$ as $m=2^{j_{1}}+2^{j_{2}}+\cdots+2^{j_{r}}$, with $0 \leqq j_{1} \leqq j_{2} \leqq \cdots \leqq j_{r}$, then a homology basis for $H_{*}\left(\Sigma_{m}\right)$ is given by elements

$$
Q_{I_{1}} * Q_{I_{2}} * \cdots * Q_{I_{r}}
$$

where $0 \leqq$ length $\left(I_{k}\right) \leqq j_{k}$, the $I_{k}$ have the same form as in 1.2 , and $\left(j_{1}, \ldots, j_{r}\right)$ range over all $r$-tuples giving a partition of $m$ as described above.

For our cohomology calculations it will be necessary to describe how some of these classes come from detecting subgroups in $\Sigma_{m}$. Inductively define $V_{n}^{\prime} \cong(Z / 2)^{n}$ by $V_{1}^{\prime}=\Sigma_{2}$ and $\left.V_{n}^{\prime}=Z / 2 \times V_{n-1}^{\prime} \subset Z / 2 \times \Sigma_{2^{n-1}} \subset Z / 2\right\} \Sigma_{2^{n-1}} \subset \Sigma_{2^{n}}$. Then, under this inclusion, the image of $H_{*}\left(V_{n}^{\prime}\right)$ is contained in the vector space spanned by the elements $Q_{l}, I=\left(i_{1}, \ldots, i_{n}\right)$ where $0 \leqq i_{1} \leqq \cdots \leqq i_{n}$. The groups $V_{n}^{\prime}$ are conjugate to the groups $V_{n}^{*}$ defined in Sect. 2 and they will play a central role in our cohomology calculations. For later use we note that the dual classes $Q_{I}^{*} \in H^{*}\left(\Sigma_{2^{n}}\right)$, where $i_{n}=1$, will restrict to generators of the image in $H^{*}\left(V_{n}\right)$ (and $H^{*}\left(V_{n}^{\prime}\right)$ ), the so-called Dickson invariants. In our calculations only the first few Dickson classes will appear; we denote them as follows: $c_{2}=\left(Q_{0,1}\right)^{*}, c_{3}=\left(Q_{1,1}\right)^{*}, d_{4}=\left(Q_{0,0,1}\right)^{*}$, $d_{6}=\left(Q_{0,1,1}\right)^{*}, d_{7}=\left(Q_{1,1,1}\right)^{*}$. 
If $m$ is not a power of two, we proceed as follows. Let $m=2^{n_{1}}+2^{n_{2}}+\cdots+2^{n_{k}}$, $0 \leqq n_{1}<n_{2}<\cdots<n_{k}$ be the dyadic expansion of $m$. Then the map induced by the natural inclusion

$$
H_{*}\left(\Sigma_{2^{n_{1}}} \times \cdots \times \Sigma_{2^{n_{k}}}\right) \rightarrow H_{*}\left(\Sigma_{m}\right)
$$

is surjective (and injective in cohomology). Now given an inclusion $i: \Sigma_{s} \times \Sigma_{t} \subset \Sigma_{s+1}$, it is clear from the definitions that $i_{*}\left(Q_{\Omega} \otimes Q_{J}\right)=Q_{I} * Q_{J}$. Taking the products of the classes originating in various detecting subgroups, we can keep track of the necessary information. This will be applied to compute the cohomology of $\Sigma_{6}, \Sigma_{8}, \Sigma_{10}$ and $\Sigma_{12}$ in Sect. 3.

Finally we point out how the symmetric classes can be described in terms of the $Q_{I}$. If $m=2 k$, then $i:\left(\Sigma_{2}\right)^{k} \hookrightarrow \Sigma_{m}$ and $i_{*}\left(Q_{1} \otimes \cdots \otimes Q_{1}\right)=Q_{1} * \cdots * Q_{1}$. Hence $i^{*}\left(\left(Q_{1} * \cdots * Q_{1}\right)^{*}\right)=\sigma_{k}$, the $k$-th symmetric class and similarly $i^{*}\left(\left(Q_{1} * 1 \cdots * 1\right)^{*}\right)=\sigma_{1}$, $i^{*}\left(\left(Q_{1} * Q_{1} * 1 * \cdots * 1\right)^{*}\right)=\sigma_{2}$, etc. Note that some of the Dickson and symmetric generators are identified: $\sigma_{2}$ with $c_{2}, \sigma_{4}$ with $d_{4}$. We use symmetric generators whenever possible.

\section{Invariant theory}

As we saw in Sect. 1, the additive structure of $H^{*}\left(\Sigma_{n}\right)$ has been completely understood for over 20 years. At that time the ring structure of $H^{*}\left(\Sigma_{4}\right)$ was determined to be the following:

$$
H^{*}\left(\Sigma_{4}\right) \cong P\left[\sigma_{1}, \sigma_{2}, c_{3}\right] /\left\langle\sigma_{1} c_{3}=0\right\rangle .
$$

It was assumed that the same type of simple relation would hold for symmetric groups of higher degree. In this section we will use invariant theory to show otherwise. In fact we will exhibit numerical evidence that indicates the presence of very complicated relations, rich with symmetry, that build up successively. We will use these to give complete descriptions of the rings $H^{*}\left(\Sigma_{n}\right)$ for $n=6,8,10,12$ in Sect. 3.

Let $\left.\left.j: V_{n}=(Z / 2)^{n} \hookrightarrow Z / 2\right\} \cdots\right\} Z / 2 \hookrightarrow \Sigma_{2^{n}}$ denote the embedding where we consider $\Sigma_{2^{n}}$ as the automorphism group of the set $\bigoplus_{1}^{n} Z / 2$ and $V_{n}$ as the set of translations. Its normalizer is the group of affine transformations, $\operatorname{Aff}_{n}(Z / 2)$. Therefore $N\left(V_{n}\right) / V_{n}=G L_{n}(\mathrm{Z} / 2)$ and we have that in fact $i m j^{*}=H^{*}\left(V_{n}\right)^{G L_{n}(\mathbf{Z} / 2)}$, the ring of invariants $(\sec [\mathrm{M}-\mathrm{M}])$. For $n=2$, we obtain that $i m j^{*} \cong P\left[x_{0}, x_{1}\right] \operatorname{deg} x_{0}=2$, $\operatorname{deg} x_{1}=3$.

For $n=4 k$, we have a natural inclusion

$$
i:\left(V_{2}\right)^{k} \hookrightarrow\left(\Sigma_{4}\right)^{k} \hookrightarrow \Sigma_{n}
$$

In cohomology, the image of $i^{*}$ lies in $\left(\bigotimes_{i=1}^{k} P\left[x_{0}, x_{1}\right]\right)^{\Sigma_{k}}$, where $\Sigma_{k}$ acts by permuting the polynomial generators. We will now analyze this ring of invariants, which later on we will see is the homomorphic image of $H^{*}\left(\Sigma_{n}\right)$.

We start with the case $k=2$ : 
Theorem 2.1. The Poincaré series of $\left(P\left[x_{0}, x_{1}\right] \otimes P\left[x_{0}, x_{1}\right]\right)^{\Sigma_{2}}$ is

$$
P_{2}(t)=\frac{1+t^{5}}{\left(1-t^{2}\right)\left(1-t^{3}\right)\left(1-t^{4}\right)\left(1-t^{6}\right)}
$$

Proof. As we are taking two-fold symmetric tensors, there are two types of invariants, $x \otimes y+y \otimes x$ and $x \otimes x$. The latter are counted by the Poincaré series $\frac{1}{\left(1-t^{4}\right)\left(1-t^{6}\right)}$. Consequently, the former are counted by the series $1 / 2\left[\frac{1}{\left(1-t^{2}\right)^{2}\left(1-t^{3}\right)^{2}}-\frac{1}{\left(1-t^{4}\right)\left(1-t^{6}\right)}\right]$ and doing an easy manipulation we obtain:

$$
\begin{aligned}
P_{2}(t) & =\frac{1}{2}\left[\left(\frac{1}{\left(1-t^{2}\right)\left(1-t^{3}\right)}\right)^{2}+\frac{1}{\left(1-t^{4}\right)\left(1-t^{6}\right)}\right] \\
& =\frac{1}{2}\left(\frac{1}{\left(1-t^{2}\right)\left(1-t^{3}\right)}\right)\left(\frac{1}{\left(1-t^{2}\right)\left(1-t^{3}\right)}+\frac{1}{\left(1+t^{2}\right)\left(1+t^{3}\right)}\right) \\
& =\frac{1}{2}\left(\frac{1}{\left(1-t^{2}\right)\left(1-t^{3}\right)}\right)\left(\frac{2+2 t^{5}}{\left(1-t^{4}\right)\left(1-t^{6}\right)}\right) \\
& =\frac{1+t^{5}}{\left(1-t^{2}\right)\left(1-t^{3}\right)\left(1-t^{4}\right)\left(1-t^{6}\right)}
\end{aligned}
$$

Examining this series, we deduce the existence of a five-dimensional class, which satisfies a quadratic relation. This is the first indication of the occurrence of relations which are not of the form seen before, the products of two generators being zero.

\section{Theorem 2.2.}

where

$$
\left(P\left[x_{0}, x_{1}\right] \otimes P\left[x_{0}, x_{1}\right]\right)^{\Sigma_{2}} \cong \frac{P\left[d_{01}, d_{02}, d_{11}, d_{22}\right]\left(x_{5}\right)}{\left\langle x_{5}^{2}+x_{5} d_{01} d_{11}+d_{22} d_{01}^{2}+d_{02} d_{11}^{2}=0\right\rangle}
$$

$$
\begin{aligned}
d_{01} & =x_{0} \otimes 1+1 \otimes x_{0}, \quad d_{11}=x_{1} \otimes 1+1 \otimes x_{1} \\
d_{02} & =x_{0} \otimes x_{0}, \quad d_{22}=x_{1} \otimes x_{1} \\
x_{5} & =x_{0} \otimes x_{1}+x_{1} \otimes x_{0} .
\end{aligned}
$$

Proof. The elements $d_{01}, d_{02}, d_{11}, d_{22}$ are independent, generating a subpolynomial algebra. Verifying the relation, we conclude that together with $x_{5}$ they generate a subalgebra with the same Poincaré series as $\left(P\left[x_{0}, x_{1}\right] \otimes P\left[x_{0}, x_{1}\right]\right)^{x_{2}}$.

The relation in 2.2 will pull back to $H^{*}\left(\Sigma_{8}\right)$, as will be shown in Sect. 3 .

Next we analyze the case $k=3$, denote $P_{3}=\left(P\left[x_{0}, x_{1}\right] \otimes P\left[x_{0}, x_{1}\right] \otimes\right.$ $\left.P\left[x_{0}, x_{1}\right]\right)^{\Sigma_{3}}$.

Theorem 2.3. The Poincaré series for $P_{3}$ is

$$
P_{3}(t)=\frac{1+t^{5}+t^{7}+t^{8}+t^{10}+t^{15}}{\left(1-t^{2}\right)\left(1-t^{3}\right)\left(1-t^{4}\right)\left(1-t^{6}\right)^{2}\left(1-t^{9}\right)}
$$


Proof. Denote

$$
\begin{aligned}
a b c & =\left(1 /\left(1-t^{2}\right)\left(1-t^{3}\right)-1\right)^{3} \\
a^{2} b & =\left(1 /\left(1-t^{4}\right)\left(1-t^{6}\right)-1\right)\left(1 /\left(1-t^{2}\right)\left(1-t^{3}\right)-1\right) \\
a^{3} & =\left(1 /\left(1-t^{6}\right)\left(1-t^{9}\right)-1\right)
\end{aligned}
$$

The letters denote the type of products they represent. Let

$$
\begin{aligned}
e_{3} & =a^{3} \leftarrow \text { cubes } \\
e_{12} & =a^{2} b-e_{3} \leftarrow \text { products of type } a^{2} b \text { which are not cubes } \\
e_{111} & =a b c-3 e_{12}-e_{3} \leftarrow \text { products of type } a b c \text { not of two other types. }
\end{aligned}
$$

As we are taking symmetric invariants, the formula for the desired Poincaré series is

$$
P_{3}(t)=\frac{1}{6} e_{111}+e_{12}+e_{3}+\left(1 /\left(1-t^{2}\right)\left(1-t^{3}\right)\right)
$$

The term on the extreme right records the 3-fold symmetrization of the generators of $P\left[x_{0}, x_{1}\right]$.

Using macsyma*, this simplifies to the asserted Poincare series.

As before, we use the numerator to deduce the relations. Let $S\left(u_{1} \otimes u_{2} \otimes u_{3}\right)$ denote symmetrization.

\section{Theorem 2.4.}

$$
P_{3} \cong P\left[d_{01}, d_{02}, d_{03}, d_{11}, d_{22}, d_{33}, x_{5}, x_{7}, x_{8}\right] /\langle R\rangle
$$

where

$$
\begin{array}{rll}
d_{01}=S\left(x_{0} \otimes 1 \otimes 1\right), & d_{02}=S\left(x_{0} \otimes x_{0} \otimes 1\right), & d_{03}=x_{0} \otimes x_{0} \otimes x_{0} \\
d_{11}=S\left(x_{1} \otimes 1 \otimes 1\right), & d_{22}=S\left(x_{1} \otimes x_{1} \otimes 1\right), & d_{33}=x_{1} \otimes x_{1} \otimes x_{1} \\
x_{5}=S\left(x_{0} \otimes x_{1} \otimes 1\right), & x_{7}=S\left(x_{0} \otimes x_{0} \otimes x_{1}\right), & x_{8}=S\left(x_{0} \otimes x_{1} \otimes x_{1}\right)
\end{array}
$$

Relations:

$$
\begin{aligned}
& x_{7}^{2}+d_{02} x_{5}^{2}+d_{02} d_{01} x_{8}+\left(d_{03} d_{11}+d_{02} d_{01} d_{11}\right) x_{5} \\
& \quad+d_{02}^{2} d_{11}^{2}+d_{01}^{2} d_{22} d_{02}+d_{03} d_{01} d_{22} \\
& x_{8}^{2}+d_{22} x_{5}^{2}+d_{22} d_{11} x_{7}+\left(d_{33} d_{01}+d_{22} d_{11} d_{01}\right) x_{5} \\
& \quad+d_{22}^{2} d_{01}^{2}+d_{11}^{2} d_{02} d_{22}+d_{33} d_{11} d_{02} \\
& x_{5} x_{7}+d_{01} x_{5}^{2}+\left[d_{01}^{2}+d_{02}\right] x_{8}+d_{01}^{2} d_{11} x_{5}+d_{01}^{3} d_{22} \\
& \quad+d_{01} d_{22} d_{02}+d_{03} d_{11}^{2}+d_{03} d_{22}+d_{01} d_{02} d_{11}^{2} \\
& x_{5} x_{8}+d_{11} x_{5}^{2}+\left[d_{11}^{2}+d_{22}\right] x_{7}+d_{11}^{2} d_{01} x_{5}+d_{11}^{3} d_{02} \\
& \quad+d_{11} d_{02} d_{22}+d_{33} d_{01}^{2}+d_{33} d_{02}+d_{11} d_{22} d_{01}^{2} \\
& x_{5}^{3}+d_{11} d_{01} x_{5}^{2}+x_{7} x_{8}+d_{11} d_{01}^{2} x_{8}+d_{01} d_{11}^{2} x_{7} \\
& +\left(d_{01}^{2} d_{22}+d_{02} d_{11}^{2}\right) x_{5}+d_{01}^{3} d_{33}+d_{11}^{3} d_{03}+d_{03} d_{33}
\end{aligned}
$$

* macsyma is a symbolic manipulation program developed at MIT and distributed by symbolics 
Proof. The elements $d_{i j}, i=0,1, j=1,2,3$ are algebraically independent, and generate a polynomial subalgebra. We examine the numerator of the Poincare series for $P_{3}$ :

$$
1+t^{5}+t^{7}+t^{8}+t^{10}+t^{15}
$$

The quintic term must come from $x_{5}=S\left(x_{0} \otimes x_{1} \otimes 1\right)$ and similarly $x_{7}=$ $S\left(x_{0} \otimes x_{0} \otimes x_{1}\right), x_{8}=S\left(x_{0} \otimes x_{1} \otimes x_{1}\right)$ account for $t^{7}, t^{8}$. Likewise $x_{5}^{2}$ explains the occurrence of $t^{10}$. (One checks that $x_{5}$ does not satisfy a quadratic equation).

There must be relations involving $x_{5}^{3}, x_{7}^{2}, x_{8}^{2}, x_{5} x_{7}$ and $x_{5} x_{8}$. Finding the exact relation is a rather tedious task, which involves taking all possible products of the right dimension involving $x_{5}, x_{7}, x_{8}, x_{5}^{2}$ and expressing their spill over in terms of the $d_{i j}$. Knowing the exact formulas, their verification is a lengthy but straight forward calculation, which we leave as a horrible exercise to the reader. Note that relation (2) is obtained from (1) by exchanging $x_{0}$ and $x_{1}$; likewise we get (4) from (3) in this manner. Relation (5) is invariant under this exchange.

To complete the proof we just compare Poincaré series.

For the cases $k=4,5,6$ we do not have the algebra structure, only the Poincare series. These can be obtained in a combinatorial way extending the methods used in 2.3; we used macsyma to perform the simplifications. As the denominators of these series are clear, we only give the numerators:

Theorem 2.5. The invariant algebras $P_{4}, P_{5}, P_{6}$ have the following polynomials as numerators of their Poincaré series:

$$
\begin{aligned}
N_{4}(t)= & t^{30}+t^{25}+t^{23}+t^{22}+t^{21}+2 t^{20}+t^{19}+t^{18}+t^{17}+t^{16}+2 t^{15}+t^{14}+t^{13} \\
& +t^{12}+t^{11}+2 t^{10}+t^{9}+t^{8}+t^{7}+t^{5}+1 \\
N_{5}(t)= & t^{50}+t^{45}+t^{43}+t^{42}+t^{41}+2 t^{40}+2 t^{39}+2 t^{38}+2 t^{37}+3 t^{36}+3 t^{35}+3 t^{34} \\
& +3 t^{33}+4 t^{32}+4 t^{31}+4 t^{30}+5 t^{29}+5 t^{28}+5 t^{27}+5 t^{26}+6 t^{25}+5 t^{24}+5 t^{23} \\
& +5 t^{22}+5 t^{21}+4 t^{20}+4 t^{19}+4 t^{18}+3 t^{17}+3 t^{16}+3 t^{15}+3 t^{14}+2 t^{13}+2 t^{12} \\
& +2 t^{11}+2 t^{10}+t^{9}+t^{8}+t^{7}+t^{5}+1 \\
N_{6}(t)= & t^{75}+t^{70}+t^{68}+t^{67}+t^{66}+2 t^{65}+2 t^{64}+2 t^{63}+3 t^{62}+4 t^{61}+4 t^{60}+5 t^{59} \\
& +5 t^{58}+6 t^{57}+7 t^{56}+8 t^{55}+10 t^{54}+10 t^{53}+11 t^{52}+13 t^{51}+14 t^{50}+15 t^{49} \\
& +17 t^{48}+18 t^{47}+19 t^{46}+20 t^{45}+21 t^{44}+22 t^{43}+23 t^{42}+23 t^{41}+24 t^{40} \\
& +23 t^{39}+24 t^{38}+24 t^{37}+23 t^{36}+24 t^{35}+23 t^{34}+23 t^{33}+22 t^{32}+21 t^{31} \\
& +20 t^{30}+19 t^{29}+18 t^{28}+17 t^{27}+15 t^{26}+14 t^{25}+13 t^{24}+11 t^{23}+10 t^{22} \\
& +10 t^{21}+8 t^{20}+7 t^{19}+6 t^{18}+5 t^{17}+5 t^{16}+4 t^{15}+4 t^{14}+3 t^{13}+2 t^{12} \\
& +2 t^{11}+2 t^{10}+t^{9}+t^{8}+t^{7}+t^{5}+1 .
\end{aligned}
$$

These polynomials satisfy two unexpected conditions: (i) if $n=\operatorname{deg} N_{i}(t)$, then the coefficient of $t^{n-k}$ is the same as the coefficient of $t^{k}$, and (ii) $N_{i}(1)=i !$.

Let $P\left[x_{1}, \ldots, x_{n}\right]$ be a polynomial algebra which has a $\Sigma_{n}$ action defined by permuting the $n$ generators. A fundamental result in Galois Theory is that the ring of invariants of this action is also a polynomial ring, on the symmetric generators $\sigma_{1}, \ldots, \sigma_{n}$. We have shown that this result does not extend to invariants 
of the form $\left(\bigotimes_{1}^{k} P\left[x_{0}, x_{1}\right]\right)^{\Sigma}$. As more variables are introduced, the complexity of this ring of invariants increases and this will be reflected by interesting relations in the cohomology of the symmetric groups.

\section{3. $H^{*}\left(\Sigma_{n}\right), n=6,8,10,12$}

In this section we will combine the well-known additive structure of $H_{*}\left(\Sigma_{n}\right)$ with the invariant theory of Sect. 2 to obtain precise descriptions of the cohomology rings $H^{*}\left(\Sigma_{n}\right) n=6,8,10,12$, as well as the action of the Steenrod algebra on them. We start by describing $H^{*}\left(\Sigma_{6}\right)$ :

\section{Theorem 3.1.}

$$
H^{*}\left(\Sigma_{6}\right) \cong P\left[\sigma_{1}, \sigma_{2}, \sigma_{3}, c_{3}\right] /\left\langle c_{3}\left(\sigma_{3}+\sigma_{1} \sigma_{2}\right)=0\right\rangle
$$

$\operatorname{deg} \sigma_{i}=i, \operatorname{deg} c_{3}=3$ where

$$
\begin{aligned}
& S q^{1} \sigma_{2}=\sigma_{1} \sigma_{2}+\sigma_{3}+c_{3}, \quad S q^{1} c_{3}=0, \quad S q^{2} c_{3}=\sigma_{2} c_{3} \\
& S q^{1} \sigma_{3}=\left(c_{3}+\sigma_{3}\right) \sigma_{1}, \quad S q^{2} \sigma_{3}=\sigma_{3} \sigma_{2}+c_{3} \sigma_{1}^{2} .
\end{aligned}
$$

Proof. From Sect. 1, we see that $H_{*}\left(\Sigma_{6}\right)$ has a basis given by elements $\left\{Q_{i} * Q_{j} * Q_{k}, Q_{r, s} * Q_{i}\right\}$ where $1 \leqq r \leqq s, \operatorname{dim} Q_{r, s}=2 s+r$ and $Q_{0, s}=Q_{s} * Q_{s^{*}}$ We now consider the sequence of inclusions

$$
\left(\Sigma_{2}\right)^{3} \stackrel{i}{\hookrightarrow} \Sigma_{4} \times \Sigma_{2} \stackrel{j}{\longrightarrow} \Sigma_{6}
$$

Using a basis dual to the one above, we have that if $k=j \cdot i$,

$$
\begin{aligned}
k^{*}\left(\left(Q_{1} * 1 * 1\right)^{*}\right) & =\sigma_{1} \\
k^{*}\left(\left(Q_{1} * Q_{1} * 1\right)^{*}\right) & =\sigma_{2} \\
k^{*}\left(\left(Q_{1} * Q_{1} * Q_{1}\right)^{*}\right) & =\sigma_{3} .
\end{aligned}
$$

Using the symbol $\sigma_{i}$ to denote the cohomology classes identified to symmetric classes, the above implies that

$$
\begin{aligned}
j^{*}\left(\left(Q_{1} * 1 * 1\right)^{*}\right) & =\sigma_{1} \otimes 1+1 \otimes \sigma_{1} \\
j^{*}\left(\left(Q_{1} * Q_{1} * 1\right)^{*}\right) & =\sigma_{1} \otimes \sigma_{1}+\sigma_{2} \otimes 1 \\
j^{*}\left(\left(Q_{1} * Q_{1} * Q_{1}\right)^{*}\right) & =\sigma_{2} \otimes \sigma_{1} .
\end{aligned}
$$

Note that the 3-dimensional generator $c_{3}$ in $H^{*}\left(\Sigma_{4}\right)$ restricts to zero. As $j_{*}\left(Q_{11} \otimes 1\right)=Q_{11} * 1$, we conclude that $j^{*}\left(\left(Q_{11} * 1\right)^{*}\right)=Q_{11} \otimes 1=c_{3} \otimes 1$.

The elements $\sigma_{1} \otimes 1+1 \otimes \sigma_{1}, \sigma_{1} \otimes \sigma_{1}+\sigma_{2} \otimes 1$ and $\sigma_{2} \otimes \sigma_{1}$ are independent, as they map to the symmetric generators under $i^{*}$. Recalling that in $H^{*}\left(\Sigma_{4}\right), c_{3} \sigma_{1}=0$ is the only relation, we obtain a unique relation in the subalgebra generated by the above elements together with $c_{3} \otimes 1$ :

$$
\left(c_{3} \otimes 1\right)\left[\left(\sigma_{2} \otimes \sigma_{1}\right)+\left(\sigma_{1} \otimes 1+1 \otimes \sigma_{1}\right)\left(\sigma_{1} \otimes \sigma_{1}+\sigma_{2} \otimes 1\right)\right]=0
$$

Hence observing that $j^{*}$ is a monomorphism and that this subalgebra has the 
same Poincaré series as $H^{*}\left(\Sigma_{6}\right)$ (which is $\left.1+x^{3} /(1-x)\left(1-x^{2}\right)\left(1-x^{3}\right)\right)$ we conclude

$$
H^{*}\left(\Sigma_{6}\right) \cong P\left[\sigma_{1}, \sigma_{2}, \sigma_{3}, c_{3}\right] /\left\langle c_{3}\left(\sigma_{3}+\sigma_{1} \sigma_{2}\right)=0\right\rangle .
$$

Here we identify $\sigma_{1} \otimes 1+1 \otimes \sigma_{1} \rightarrow \sigma_{1}, \quad \sigma_{1} \otimes \sigma_{1}+\sigma_{2} \otimes 1 \rightarrow \sigma_{2}, \quad \sigma_{2} \otimes \sigma_{1} \rightarrow \sigma_{3}$, $c_{3} \otimes 1 \rightarrow c_{3}$.

For the action of the Steenrod algebra, we apply the $S q^{i}$ in $H^{*}\left(\Sigma_{4}\right) \otimes H^{*}\left(\Sigma_{2}\right)$ and use the relations there:

$$
\begin{aligned}
S q^{1} \sigma_{2}= & S q^{1} i^{*}\left(\sigma_{2} \otimes 1+\sigma_{1} \otimes \sigma_{1}\right)=i^{*}\left(S q^{1} \sigma_{2} \otimes 1+\sigma_{1}^{2} \otimes \sigma_{1}+\sigma_{1} \otimes \sigma_{1}^{2}\right) \\
& =i^{*}\left(c_{3} \otimes 1+\sigma_{1} \sigma_{2} \otimes 1+\sigma_{1}^{2} \otimes \sigma_{1}+\sigma_{1} \otimes \sigma_{1}^{2}\right) \\
& =i^{*}\left(\left(\sigma_{1} \otimes 1+1 \otimes \sigma_{1}\right)\left(\sigma_{1} \otimes \sigma_{1}+\sigma_{2} \otimes 1\right)+\sigma_{2} \otimes \sigma_{1}+c_{3} \otimes 1\right) \\
& =\sigma_{1} \sigma_{2}+\sigma_{3}+c_{3} \\
S q^{1} c_{3}= & S q^{1} i^{*}\left(c_{3} \otimes 1\right)=i^{*}\left(S q^{1}\left(c_{3} \otimes 1\right)\right)=0 \\
S q^{2} c_{3}= & i^{*}\left(S q^{2}\left(c_{3} \otimes 1\right)\right)=i^{*}\left(\sigma_{2} C_{3} \otimes 1\right)=\sigma_{2} c_{3} \\
S q^{1} \sigma_{3}= & S q^{1} i^{*}\left(\sigma_{2} \otimes \sigma_{1}\right)=i^{*}\left(S q^{1} \sigma_{2} \otimes \sigma_{1}+\sigma_{2} \otimes \sigma_{1}^{2}\right) \\
& =i^{*}\left(c_{3} \otimes \sigma_{1}+\sigma_{1} \sigma_{2} \otimes \sigma_{1}+\sigma_{2} \otimes \sigma_{1}^{2}\right) \\
& =\left(c_{3}+\sigma_{3}\right) \sigma_{1} \\
S q^{2} \sigma_{3}= & i^{*}\left(S q^{2} \sigma_{2} \otimes \sigma_{1}+S q^{1} \sigma_{2} \otimes S q^{1} \sigma_{1}\right)=i^{*}\left(\sigma_{2}^{2} \otimes \sigma_{1}+c_{3} \otimes \sigma_{1}^{2}+\sigma_{1} \sigma_{2} \otimes \sigma_{1}^{2}\right) \\
= & i^{*}\left(\left(\sigma_{1} \otimes \sigma_{1}\right)\left(\sigma_{2} \otimes 1+\sigma_{1} \otimes \sigma_{1}\right)+\left(c_{3} \otimes 1\right)\left(\sigma_{1} \otimes 1+1 \otimes \sigma_{1}\right)^{2}\right) \\
& =\sigma_{3} \sigma_{2}+c_{3} \sigma_{1}^{2} .
\end{aligned}
$$

Next we consider $\Sigma_{8}$ :

\section{Theorem 3.2.}

$$
H^{*}\left(\Sigma_{8}\right) \cong P\left[\sigma_{1}, \sigma_{2}, \sigma_{3}, c_{3}, \sigma_{4}, d_{6}, d_{7}\right]\left(x_{5}\right) /\langle R\rangle
$$

where $\operatorname{deg} \sigma_{i}=i, \operatorname{deg} c_{3}=3, \operatorname{deg} d_{i}=i, \operatorname{deg} x_{5}=5$, and $R$ is the following set of relations:

$$
\begin{aligned}
& d_{6} \sigma_{1}=d_{6} \sigma_{3}=0 \\
& d_{7} \sigma_{1}=d_{7} \sigma_{2}=d_{7} \sigma_{3}=d_{7} c_{3}=d_{7} x_{5}=0 \\
& x_{5} \sigma_{3}+c_{3} \sigma_{4} \sigma_{1}=0 \\
& c_{3}\left(\sigma_{3}+\sigma_{1} \sigma_{2}\right)+\sigma_{1} x_{5}=0 \\
& x_{5}^{2}+x_{5} \sigma_{2} c_{3}+d_{6} \sigma_{2}^{2}+\sigma_{4} c_{3}^{2}=0 .
\end{aligned}
$$

The following table describe the action of the Steenrod algebra:

\begin{tabular}{cccc}
\hline & $\sigma_{2}$ & $\sigma_{3}$ & $c_{3}$ \\
\hline$S q^{1}$ & $\sigma_{1} \sigma_{2}+\sigma_{3}+c_{3}$ & $\sigma_{1}\left(c_{3}+\sigma_{3}\right)$ & 0 \\
\hline$S q^{2}$ & $\sigma_{2}^{2}$ & $c_{3} \sigma_{1}^{2}+\sigma_{2} \sigma_{3}+\sigma_{1} \sigma_{4}$ & $\sigma_{2} c_{3}+x_{5}$ \\
\hline$S q^{3}$ & 0 & $\sigma_{3}^{2}$ & $c_{3}^{2}$ \\
\hline
\end{tabular}




\begin{tabular}{ccccc}
\hline & $\sigma_{4}$ & $x_{5}$ & $d_{6}$ & $d_{7}$ \\
\hline$S q^{1}$ & $x_{5}+\sigma_{1} \sigma_{4}$ & $\sigma_{1} x_{5}$ & $d_{7}$ & 0 \\
\hline$S q^{2}$ & $\sigma_{2} \sigma_{4}+d_{6}+x_{5} \sigma_{1}$ & $\sigma_{2} x_{5}$ & $\sigma_{2} d_{6}$ & 0 \\
\hline$S q^{3}$ & $d_{7}+c_{3} \sigma_{4}+\sigma_{3} \sigma_{4}+\sigma_{2} x_{5}$ & $\left(c_{3}+\sigma_{3}\right) x_{5}$ & $c_{3} d_{6}$ & 0 \\
\hline$S q^{4}$ & $\sigma_{4}^{2}$ & $\sigma_{4} x_{5}+c_{3}\left(d_{6}+\sigma_{1} x_{5}\right)$ & $\sigma_{4} d_{6}$ & $\sigma_{4} d_{7}$ \\
\hline$S q^{5}$ & 0 & $x_{5}^{2}$ & $x_{5} d_{6}+\sigma_{4} d_{7}$ & 0 \\
\hline$S q^{6}$ & 0 & 0 & $d_{6}^{2}$ & $d_{5} d_{7}$
\end{tabular}

Proof. We will make use of the following inclusions

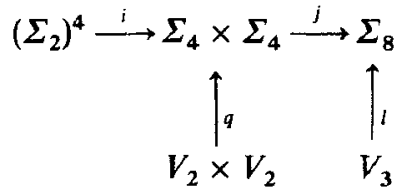

Recalling facts from Sect. 1, we see that $H_{*}\left(\Sigma_{8}\right)$ has a basis given by the elements

$$
\begin{array}{llll}
Q_{i, j, k} & \operatorname{dim} i+2 j+4 k, & 0 \leqq i \leqq j \leqq k & \\
Q_{i, j} * Q_{r} * Q_{s} & \operatorname{dim} i+2 j+r+s, & 0 \leqq i \leqq j & \\
Q_{i, j} * Q_{r, s} & \operatorname{dim} i+r+2(j+s), & 0 \leqq i \leqq j, & 0 \leqq r \leqq s \\
Q_{i} * Q_{j} * Q_{k} * Q_{s} & \operatorname{dim} i+j+k+s & &
\end{array}
$$

with relations $Q_{0, j, k}=Q_{j, k} * Q_{j, k}, Q_{0, k}=Q_{k} * Q_{k}$. As before we know the cohomology classes which map to the symmetric generators in $H^{*}\left(\left(\Sigma_{2}\right)^{4}\right)$, and what classes they represent in $H^{*}\left(\Sigma_{4}\right) \otimes H^{*}\left(\Sigma_{4}\right)$ under $j^{*}$ :

$$
\begin{aligned}
j^{*}\left(\sigma_{1}\right)=j^{*}\left(\left(Q_{1} * 1 * 1 * 1\right)^{*}\right) & =\sigma_{1} \otimes 1+1 \otimes \sigma_{1} \\
j^{*}\left(\sigma_{2}\right)=j^{*}\left(\left(Q_{1} * Q_{1} * 1 * 1\right)^{*}\right) & =\sigma_{2} \otimes 1+\sigma_{1} \otimes \sigma_{1}+1 \otimes \sigma_{2} \\
j^{*}\left(\sigma_{3}\right)=j^{*}\left(\left(Q_{1} * Q_{1} * Q_{1} * 1\right)^{*}\right) & =\sigma_{2} \otimes \sigma_{1}+\sigma_{1} \otimes \sigma_{2} \\
j^{*}\left(\sigma_{4}\right)=j^{*}\left(\left(Q_{1} * Q_{1} * Q_{1} * Q_{1}\right)^{*}\right) & =\sigma_{2} \otimes \sigma_{2} .
\end{aligned}
$$

We also have that

$$
\begin{aligned}
j_{*}\left(Q_{1,1} \otimes Q_{1} * 1\right) & =Q_{1,1} * Q_{1} * 1 \\
j_{*}\left(Q_{1,1} \otimes Q_{1} * Q_{1}\right) & =Q_{1,1} * Q_{1} * Q_{1} \\
j_{*}\left(Q_{1,1} \otimes Q_{1,1}\right) & =Q_{1,1} * Q_{1,1} .
\end{aligned}
$$

Hence in cohomology we deduce, that

$$
\begin{aligned}
j^{*}\left(\left(Q_{1,1} * Q_{1} * 1\right)^{*}\right) & =c_{3} \otimes \sigma_{1}+\sigma_{1} \otimes c_{3} \\
j^{*}\left(x_{5}\right)=j^{*}\left(\left(Q_{1,1} * Q_{1} * Q_{1}\right)^{*}\right) & =c_{3} \otimes \sigma_{2}+\sigma_{2} \otimes c_{3} \\
j^{*}\left(d_{6}\right)=j^{*}\left(\left(Q_{1,1} * Q_{1,1}\right)^{*}\right) & =c_{3} \otimes c_{3} .
\end{aligned}
$$


We also have $j^{*}\left(c_{3}\right)=j^{*}\left(\left(Q_{1,1} * 1\right)^{*}\right)=c_{3} \otimes 1+1 \otimes c_{3}$ and $j^{*}\left(d_{7}\right)=j^{*}\left(\left(Q_{1,1,1}\right)^{*}\right)=0$. Note that we have renamed our cohomology generators in such a way as to keep track of the symmetric generators $\left(\sigma_{i}\right)$ and the symmetrization of the three dimensional generator of $H^{*}\left(\Sigma_{4}\right), c_{3}$.

To determine one of the multiplicative relations we use Theorem 2.2 and the fact that $q^{*} j^{*}$ is clearly onto the invariants previously calculated; in fact we have $(j q)^{*}\left(\sigma_{2}\right)=d_{01},(j q)^{*}\left(c_{3}\right)=d_{11},(j q)^{*}\left(\sigma_{4}\right)=d_{02},(j q)^{*}\left(d_{6}\right)=d_{11},(j q)^{*}\left(x_{5}\right)=x_{5}$, and the other generators map to 0 . From 2.2 we pull back the relation to obtain $j^{*}\left(x_{5}^{2}+x_{5} \sigma_{2} c_{3}+d_{6} \sigma_{2}^{2}+\sigma_{4} c_{3}^{2}\right)=0$. We now make use of the fact that $i m\left(l^{*}\right)$ is well known $[\mathrm{M}-\mathrm{M}]$ to be a polynomial algebra on the Dickson invariants $D_{4}, D_{6}, D_{7}$, (deg $\left.D_{i}=i\right)$. Furthermore it is known that $\left.l^{*}\left(Q_{1} * Q_{1} * Q_{1} * Q_{1}\right)^{*}\right)=l^{*}\left(\sigma_{4}\right)=D_{4}$, $l^{*}\left(\left(Q_{1,1} * Q_{1,1}\right)^{*}\right)=l^{*}\left(d_{6}\right)=D_{6}, l^{*}\left(\left(Q_{1,1,1}\right)^{*}\right)=l^{*}\left(d_{7}\right)=D_{7}$ and all other generators map to 0 .

It was also proved in [M-M] that $V_{3}$ and $\Sigma_{4} \times \Sigma_{4}$ are detecting subgroups for $\Sigma_{8}$. In other words, $H^{*}\left(\Sigma_{8}\right)$ is isomorphic to the subalgebra of $H^{*}\left(\Sigma_{4} \times \Sigma_{4}\right) \oplus H^{*}\left(V_{3}\right)$ generated by

$$
\begin{aligned}
& \sigma_{1} \mapsto\left(\sigma_{1} \otimes 1+1 \otimes \sigma_{1}, 0\right) \\
& \sigma_{2} \mapsto\left(\sigma_{2} \otimes 1+\sigma_{1} \otimes \sigma_{1}+1 \otimes \sigma_{2}, 0\right) \\
& \sigma_{3} \mapsto\left(\sigma_{2} \otimes \sigma_{1}+\sigma_{1} \otimes \sigma_{2}, 0\right) \\
& \sigma_{4} \mapsto\left(\sigma_{2} \otimes \sigma_{2}, D_{4}\right) \\
& c_{3} \mapsto\left(c_{3} \otimes 1+1 \otimes c_{3}, 0\right) \\
& x_{5} \mapsto\left(c_{3} \otimes \sigma_{2}+\sigma_{2} \otimes c_{3}, 0\right) \\
& d_{6} \mapsto\left(c_{3} \otimes c_{3}, D_{6}\right) \\
& d_{7} \mapsto\left(0, D_{7}\right) .
\end{aligned}
$$

The relations

$$
d_{6} \sigma_{1}=d_{6} \sigma_{3}=d_{7} \sigma_{1}=d_{7} \sigma_{3}=d_{7} \sigma_{2}=d_{7} c_{3}=d_{7} x_{5}=0
$$

follow immediately from this, as does the last relation (using 2.2). Now, under this correspondence

$$
\begin{aligned}
& x_{5} \sigma_{3}+c_{3} \sigma_{4} \sigma_{1} \mapsto\left(\left(c_{3} \otimes \sigma_{2}+\sigma_{2} \otimes c_{3}\right)\left(\sigma_{2} \otimes \sigma_{1}+\sigma_{1} \otimes \sigma_{2}\right), 0\right) \\
&+\left(\left(c_{3} \otimes 1+1 \otimes c_{3}\right)\left(\sigma_{2} \otimes \sigma_{2}\right)\left(\sigma_{1} \otimes 1+1 \otimes \sigma_{1}\right), 0\right) \\
&=\left(c_{3} \sigma_{2} \otimes \sigma_{1} \sigma_{1}+\sigma_{2} \sigma_{1} \otimes c_{3} \sigma_{2}, 0\right)+\left(c_{3} \sigma_{2} \otimes \sigma_{2} \sigma_{1}+\sigma_{2} \sigma_{1} \otimes c_{3} \sigma_{2}, 0\right) \\
&=(0,0) \\
& c_{3}\left(\sigma_{3}+\sigma_{1} \sigma_{2}\right)+\sigma_{1} x_{5} \mapsto\left(( c _ { 3 } \otimes 1 + 1 \otimes c _ { 3 } ) \left[\left(\sigma_{2} \otimes \sigma_{1}+\sigma_{1} \otimes \sigma_{2}\right)\right.\right. \\
&\left.\left.+\left(\sigma_{1} \otimes 1+1 \otimes \sigma_{1}\right)\left(\sigma_{2} \otimes 1+\sigma_{1} \otimes \sigma_{1}+1 \otimes \sigma_{2}\right)\right], 0\right) \\
&+\left(\left(\sigma_{1} \otimes 1+1 \otimes \sigma_{1}\right)\left(c_{3} \otimes \sigma_{2}+\sigma_{2} \otimes c_{3}\right), 0\right) \\
&=\left(c_{3} \sigma_{2} \otimes \sigma_{1}+\sigma_{1} \otimes c_{3} \sigma_{2}+c_{3} \sigma_{2} \otimes \sigma_{1}\right. \\
&\left.+c_{3} \otimes \sigma_{1} \sigma_{2}+\sigma_{1} \otimes c_{3} \sigma_{2}+\sigma_{1} \sigma_{2} \otimes c_{3}, 0\right) \\
&+\left(c_{3} \otimes \sigma_{1} \sigma_{2}+\sigma_{1} \sigma_{2} \otimes c_{3}, 0\right)=(0,0)
\end{aligned}
$$


The fact that this is a complete description of $H^{*}\left(\Sigma_{8}\right)$ follows from checking its Poincare series (which is known from the results in Sect. 1) or by observing that any other relation would be detected on $\left(\Sigma_{2}\right)^{4}, V_{2} \times V_{2}$ or $V_{3}$.

To obtain the action of the Steenrod algebra, we work in $\operatorname{im}\left(j^{*} \oplus l^{*}\right)$ and use our knowledge about the operations in $H^{*}\left(\Sigma_{4}\right) \otimes H^{*}\left(\Sigma_{4}\right)$ and $H^{*}\left(V_{3}\right)$. We leave the details to the reader.

Corollary 3.3. The Poincaré series of $\Sigma_{8}$ is

$P_{\Sigma_{8}}(t)$

$$
=\frac{t^{15}+2 t^{13}+t^{12}+3 t^{11}+3 t^{10}+3 t^{9}+4 t^{8}+4 t^{7}+4 t^{6}+3 t^{5}+3 t^{4}+t^{3}+2 t^{2}+1}{\left(t^{7}-1\right)\left(t^{6}-1\right)\left(t^{3}-1\right)(t-1)\left(t^{2}+1\right)} .
$$

From this, a relatively direct calculation gives the cohomology of $\Sigma_{10}$. Indeed, going from $\Sigma_{4 i}$ to $\Sigma_{4 i+2}$ only has the effect of removing relations involving $\sigma_{1}$. In general, the relations in a finite symmetric group do not occur stably, but appear at the finite stage because the detecting subgroups are not yet large enough. For $\Sigma_{10}$ we have:

\section{Theorem 3.4.}

$$
H^{*}\left(\Sigma_{10}\right) \cong P\left[\sigma_{1}, \sigma_{2}, \sigma_{3}, \sigma_{4}, \sigma_{5}, c_{3}, x_{5}, d_{6}, d_{7}\right] /\langle R\rangle
$$

where $R$ is the set of relations

$$
\begin{aligned}
& \sigma_{2} d_{7}, \sigma_{3} d_{7}, c_{3} d_{7}, x_{5} d_{7} \\
& \left(\sigma_{5}+\sigma_{1} \sigma_{4}\right) d_{7},\left(\sigma_{3}+\sigma_{1} \sigma_{2}\right) d_{6},\left(\sigma_{5}+\sigma_{1} \sigma_{4}\right) d_{6} \\
& x_{5}^{2}+\sigma_{2} c_{3} x_{5}+c_{3}^{2} \sigma_{4}+\sigma_{2}^{2} d_{6} \\
& \left(\sigma_{3}+\sigma_{1} \sigma_{2}\right) x_{5}+c_{3}\left(\sigma_{5}+\sigma_{1} \sigma_{4}+\sigma_{2} \sigma_{3}+\sigma_{1} \sigma_{2}^{2}\right) \\
& \left(\sigma_{5}+\sigma_{1} \sigma_{4}\right) x_{5}+c_{3}\left(\sigma_{3}+\sigma_{1} \sigma_{2}\right) \sigma_{4} \cdot \square
\end{aligned}
$$

Next we prove

Theorem 3.5.

$$
H^{*}\left(\Sigma_{12}\right) \cong P\left[\sigma_{1}, \sigma_{2}, \sigma_{3}, c_{3}, \sigma_{4}, \sigma_{5}, \sigma_{6}, d_{6}, d_{7}, d_{9}\right]\left(x_{5}, x_{7}, x_{8}\right) /\langle R\rangle
$$

where $\operatorname{deg} \sigma_{i}=i, \operatorname{deg} c_{3}=3, \operatorname{deg} d_{i}=i, \operatorname{deg} x_{i}=i$ and $R$ is the set of relations

$$
\begin{aligned}
& x_{7}^{2}+\sigma_{4} x_{5}^{2}+\sigma_{2} \sigma_{4} x_{8}+\left(\sigma_{6} c_{3}+\sigma_{2} \sigma_{4} c_{3}\right) x_{5}+\sigma_{4}^{2}+c_{3}^{2}+\sigma_{2}^{2} \sigma_{4} d_{6}+\sigma_{6} \sigma_{2} d_{6}, \\
& x_{8}^{2}+d_{6} x_{5}^{2}+c_{3} d_{6} x_{7}+\left(d_{9} \sigma_{2}+c_{3} d_{6} \sigma_{2}\right) x_{5}+d_{6}^{2} \sigma_{2}^{2}+c_{3}^{2} \sigma_{4} d_{6}+d_{9} c_{3} \sigma_{2}, \\
& x_{5} x_{7}+\sigma_{2} x_{5}^{2}+\left[\sigma_{2}^{2}+\sigma_{4}\right] x_{8}+\sigma_{2}^{2} c_{3} x_{5}+\sigma_{2}^{3} d_{6}+\sigma_{2} d_{6} \sigma_{4}+\sigma_{6} c_{3}^{2}+\sigma_{6} d_{6}+\sigma_{2} \sigma_{4} c_{3}^{2}, \\
& x_{5} x_{8}+c_{3} x_{5}^{2}+\left[c_{3}^{2}+d_{6}\right] x_{7}+c_{3}^{2} \sigma_{2} x_{5}+c_{3}^{3} \sigma_{4}+c_{3} \sigma_{4} d_{6}+d_{9} \sigma_{2}^{2}+d_{9} \sigma_{4}+c_{3} d_{6} \sigma_{2}^{2}, \\
& x_{5}^{3}+c_{3} \sigma_{2} x_{5}^{2}+x_{7} x_{8}+c_{3} \sigma_{2}^{2} x_{8}+\sigma_{2} c_{3}^{2} x_{7}+\left(\sigma_{2}^{2} d_{6}+\sigma_{4} c_{3}^{2}\right) x_{5}+\sigma_{2}^{3} d_{9}+c_{3}^{3} \sigma_{6}+\sigma_{6} d_{9}, \\
& d_{9} \sigma_{1}, d_{9} \sigma_{3}, d_{9} \sigma_{5} \\
& d_{7} \sigma_{3}, d_{7} x_{5}, d_{7} \sigma_{1} c_{3}, d_{7}\left(x_{7}+\sigma_{4} c_{3}\right), d_{7}\left(\sigma_{5}+\sigma_{4} \sigma_{1}\right), d_{7}\left(\sigma_{6}+\sigma_{4} \sigma_{2}\right), d_{7}\left(x_{8}+d_{6} \sigma_{2}\right), \\
& d_{7}\left(d_{9}+d_{6} c_{3}\right),
\end{aligned}
$$




$$
\begin{aligned}
& x_{7} \sigma_{1}+x_{5}\left(\sigma_{1} \sigma_{2}+\sigma_{3}\right)+c_{3}\left(\sigma_{2} \sigma_{3}+\sigma_{2}^{2} \sigma_{1}+\sigma_{1} \sigma_{4}+\sigma_{5}\right), \\
& x_{7} \sigma_{3}+x_{5}\left(\sigma_{5}+\sigma_{1} \sigma_{4}\right)+c_{3}\left(\sigma_{1} \sigma_{6}+\sigma_{3} \sigma_{4}+\sigma_{1} \sigma_{2} \sigma_{4}\right), \\
& x_{7} \sigma_{5}+x_{5} \sigma_{1} \sigma_{6}+c_{3}\left(\sigma_{3} \sigma_{6}+\sigma_{1} \sigma_{2} \sigma_{6}\right), \\
& x_{8} \sigma_{1}+d_{6}\left(\sigma_{3}+\sigma_{1} \sigma_{2}\right), \\
& x_{8} \sigma_{3}+d_{6}\left(\sigma_{5}+\sigma_{1} \sigma_{4}\right), \\
& x_{8} \sigma_{5}+d_{6} \sigma_{1} \sigma_{6} .
\end{aligned}
$$

The action on the Steenrod algebra on $H^{*}\left(\Sigma_{12}\right)$ is determined by the following values:

$$
\begin{aligned}
& S q^{1} \sigma_{2}=\sigma_{1} \sigma_{2}+\sigma_{3}+c_{3}, \\
& S q^{1} c_{3}=0, S q^{2} c_{3}=\sigma_{2} c_{3}+x_{5} \\
& S q^{2} \sigma_{3}=c_{3} \sigma_{1}^{2}+\sigma_{2} \sigma_{3}+\sigma_{1} \sigma_{4}+\sigma_{5} \\
& S q^{1} \sigma_{4}=x_{5}+\sigma_{1} \sigma_{4}+\sigma_{5}, S q^{2} \sigma_{4}=\sigma_{2} \sigma_{4}+\sigma_{6}+d_{6}+c_{3}\left(\sigma_{3}+\sigma_{1} \sigma_{2}\right) \\
& S q^{2} \sigma_{5}=d_{6} \sigma_{1}+x_{5} \sigma_{1}^{2}+c_{3}\left(\sigma_{1} \sigma_{3}+\sigma_{1}^{2} \sigma_{2}\right)+\sigma_{2} \sigma_{5}+\sigma_{1} \sigma_{6} \\
& S q^{4} \sigma_{5}=c_{3} \sigma_{3}^{2}+\sigma_{4} \sigma_{5}+\sigma_{1} x_{5} \sigma_{3}+\sigma_{3} \sigma_{6}+c_{3} \sigma_{1} \sigma_{2} \sigma_{3}+c_{3} \sigma_{1} \sigma_{5}, \\
& S q^{2} x_{5}=\sigma_{2} x_{5}, \\
& S q^{1} \sigma_{6}=x_{7}+\sigma_{1} \sigma_{6}, S q^{2} \sigma_{6}=x_{8}+x_{7} \sigma_{1}+\sigma_{2} \sigma_{6} \\
& S q^{4} \sigma_{6}=\sigma_{4} \sigma_{6}+x_{7} \sigma_{3}+d_{6} \sigma_{2}^{2}+x_{5}^{2}+x_{5} \sigma_{2} c_{3}+c_{3}^{2} \sigma_{4}+c_{3} x_{7}+c_{3} \sigma_{1} \sigma_{6}, \\
& S q^{1} d_{6}=d_{7}, S q^{2} d_{6}=x_{8}+\sigma_{2} d_{6} \\
& S q^{4} d_{6}=\sigma_{4} \sigma_{6}+x_{5} \sigma_{2} c_{3}+d_{6} \sigma_{2}^{2}+\sigma_{4} c_{3}^{2}+x_{5}^{2}+x_{7} c_{3}+x_{8} \sigma_{2}, \\
& S q^{1} d_{7}=S q^{2} d_{7}=0, S q^{4} d_{7}=\sigma_{4} d_{7}, S q^{6} d_{7}=d_{6} d_{7}, \\
& S q^{1} x_{8}=d_{9}+\sigma_{2} d_{7}+\sigma_{3} d_{6}+\sigma_{1} \sigma_{2} d_{6}, S q^{4} x_{8}=x_{8}\left(\sigma_{4}+c_{3} \sigma_{1}\right) \\
& S q^{1} d_{9}=d_{7} c_{3}, S q^{2} d_{9}=\sigma_{2} d_{9}, S q^{8} d_{9}=d_{7} x_{7} c_{3}+x_{8} d_{9} .
\end{aligned}
$$

Proof. From Sect. 1, we see that $H_{*}\left(\Sigma_{12}\right)$ has a basis given by elements

$$
\begin{array}{lll}
\text { Generators } & \text { Dimensions } & \text { Restrictions } \\
Q_{i, j, k} * Q_{r, s} & i+r+2(j+s)+4 k & 0 \leqq i \leqq j \leqq k \\
& & 0 \leqq r \leqq s \\
Q_{i, j, k} * Q_{r} * Q_{s} & i+r+s+2 j+4 k & 0 \leqq i \leqq j \leqq k \\
Q_{i, j} * Q_{r, s} * Q_{t, u} & i+r+t+2(j+s+u) & 0 \leqq i \leqq j \\
& & 0 \leqq r \leqq s \\
Q_{i, j} * Q_{r, s} * Q_{t} * Q_{u} & i+r+t+u+2(j+s) & 0 \leqq i \leqq j \\
Q_{i, j} * Q_{r} * Q_{s} * Q_{t} * Q_{u} & i+r+s+t+u+2 j & 0 \leqq r \leqq s \\
Q_{i} * Q_{j} * Q_{r} * Q_{s} * Q_{t} * Q_{k} & i+j+r+s+t+u &
\end{array}
$$


We make use of the following sequence of inclusions:

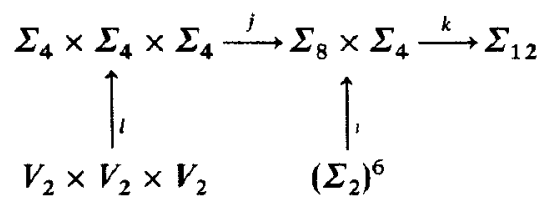

As before if we denote

$$
\begin{array}{ll}
\sigma_{1}=\left(Q_{1} * 1 * 1 * 1 * 1 * 1\right)^{*}, & \sigma_{2}=\left(Q_{1} * Q_{1} * 1 * 1 * 1 * 1\right)^{*} \\
\sigma_{3}=\left(Q_{1} * Q_{1} * Q_{1} * 1 * 1 * 1\right)^{*}, & \sigma_{4}=\left(Q_{1} * Q_{1} * Q_{1} * Q_{1} * 1 * 1\right)^{*} \\
\sigma_{5}=\left(Q_{1} * Q_{1} * Q_{1} * Q_{1} * Q_{1} * 1\right)^{*}, & \sigma_{6}=\left(Q_{1} * Q_{1} * Q_{1} * Q_{1} * Q_{1} * Q_{1}\right)^{*}
\end{array}
$$

then under $(k j i)^{*}$ they map to the symmetric generators (having the same name) in $H^{*}\left(\left(\Sigma_{2}\right)^{6}\right)$. Hence under $k^{*}$ we have

$$
\left\{\begin{array}{l}
k^{*}\left(\sigma_{1}\right)=\sigma_{1} \otimes 1+1 \otimes \sigma_{1}, \quad k^{*}\left(\sigma_{2}\right)=\sigma_{2} \otimes 1+\sigma_{1} \otimes \sigma_{1}+1 \otimes \sigma_{2} \\
k^{*}\left(\sigma_{3}\right)=\sigma_{3} \otimes 1+\sigma_{2} \otimes \sigma_{1}+\sigma_{1} \otimes \sigma_{2}, \quad k^{*}\left(\sigma_{4}\right)=\sigma_{4} \otimes 1+\sigma_{2} \otimes \sigma_{2}+\sigma_{3} \otimes \sigma_{1} \\
k^{*}\left(\sigma_{5}\right)=\sigma_{4} \otimes \sigma_{1}+\sigma_{3} \otimes \sigma_{2}, \quad k^{*}\left(\sigma_{6}\right)=\sigma_{4} \otimes \sigma_{2}
\end{array}\right.
$$

We also have

$$
\begin{aligned}
(k \circ j)_{*}\left(Q_{1,1} \otimes 1 \otimes 1\right) & =Q_{1,1} * 1 * 1 * 1 * 1 \\
(k \circ j)_{*}\left(Q_{1,1} \otimes Q_{1,1} \otimes 1\right) & =Q_{1,1} * Q_{1,1} * 1 * 1 \\
(k \circ j)_{*}\left(Q_{1,1} \otimes Q_{1,1} \otimes Q_{1,1}\right) & =Q_{1,1} * Q_{1,1} * Q_{1,1}
\end{aligned}
$$

Denoting the duals of these homology classes by $c_{3}, d_{6}, d_{9}$ respectively, and $d_{7}=\left(Q_{1,1,1} * 1 * 1\right)^{*}$ we have that

$$
\left\{\begin{array}{l}
k^{*}\left(c_{3}\right)=c_{3} \otimes 1+1 \otimes c_{3} \\
k^{*}\left(d_{6}\right)=d_{6} \otimes 1+c_{3} \otimes c_{3} \\
k^{*}\left(d_{7}\right)=d_{7} \otimes 1 \\
k^{*}\left(d_{9}\right)=d_{6} \otimes c_{3}
\end{array}\right.
$$

Similarly,

$$
\begin{gathered}
(k \circ j)_{*}\left(Q_{1,1} \otimes Q_{1} * Q_{1} \otimes 1\right)=Q_{1,1} * Q_{1} * Q_{1} * 1 * 1 \\
(k \circ j)_{*}\left(Q_{1,1} \otimes Q_{1} * Q_{1} \otimes Q_{1} * Q_{1}\right)=Q_{1,1} * Q_{1} * Q_{1} * Q_{1} * Q_{1} \\
(k \circ j)_{*}\left(Q_{1,1} \otimes Q_{1,1} \otimes Q_{1} * Q_{1}\right)=Q_{1,1} * Q_{1,1} * Q_{1} * Q_{1}
\end{gathered}
$$

Denoting the duals of these homology classes $x_{5}, x_{7}, x_{8}$ respectively, we have that

$$
\left\{\begin{array}{l}
k^{*}\left(x_{5}\right)=x_{5} \otimes 1+\sigma_{2} \otimes c_{3}+c_{3} \otimes \sigma_{2}+c_{3} \sigma_{1} \otimes \sigma_{1} \\
k^{*}\left(x_{7}\right)=\sigma_{4} \otimes c_{3}+x_{5} \otimes \sigma_{2} \\
k^{*}\left(x_{8}\right)=x_{5} \otimes c_{3}+d_{6} \otimes \sigma_{2}
\end{array}\right.
$$

The image of $k^{*}$ is generated by the 13 classes we have listed; this follows from the detecting subgroups. As $k^{*}$ is injective (note that $\Sigma_{12}$ and $\Sigma_{8} \times \Sigma_{4}$ have the 
same 2-Sylow subgroup) we conclude that $H^{*}\left(\Sigma_{12}\right)$ is isomorphic to the subalgebra of $H^{*}\left(\Sigma_{8} \times \Sigma_{4}\right)$ generated by the classes defined in (3.5), (3.6), (3.7).

To obtain the relations, we observe that there are three distinct types of generators

Type I: $\sigma_{1}, \sigma_{2}, \sigma_{3}, \sigma_{4}, \sigma_{5}, \sigma_{6}$

Type II: $\sigma_{2}, c_{3}, \sigma_{4}, \sigma_{6}, d_{6}, x_{5}, x_{7}, x_{8}, d_{9}$

Type III: $d_{7}$

The relations among the generators of type II are detected on $\left(V_{2}\right)^{3}$. The relations obtained in the ring of invariants $P_{3}$ correspond to them, and using 2.4 we pull them back to $H^{*}\left(\Sigma_{12}\right)$, yielding the first block of relations. The other relations come from products of generators of different type. They are mixed products which vanish when restricted to the detecting subgroups. We leave the verification of the relations to the reader. Note that they are consistent with the relations recorded in the symmetric groups of lower degree, and that only the new generators of type II produce new relations when multiplied with the odd dimensional symmetric generators. The fact that these are a complete set of relations follows by observing that any other relation must necessarily decompose into a combination of those given.

Finally, the action of the Steenrod algebra is determined by working in $H^{*}\left(\Sigma_{8} \times \Sigma_{4}\right)$ and using our knowledge of the action on $H^{*}\left(\Sigma_{8}\right)$ and $H^{*}\left(\Sigma_{4}\right)$.

\section{The cohomology of alternating groups}

An important class of simple groups whose cohomology has not been computed are the alternating groups $A_{n}$. The absence of proper normal subgroups makes the cohomology of simple groups particularly inaccessible, but in our situation we can make good use of $H^{*}\left(\Sigma_{n}\right)$. The following lemma in Quillen-Venkov [Q-V] allows one to relate the cohomology of a group to that of an index 2 subgroup:

Lemma 4.1. Let $v \in \operatorname{Hom}(G, \mathbf{Z} / 2)$ non-trivial, with $H=\operatorname{ker} v$. Then there is a long exact sequence

$$
\cdots \longrightarrow H^{i}(G) \stackrel{\cup v}{\longrightarrow} H^{i+1}(G) \stackrel{\text { res }}{\longrightarrow} H^{i+1}(H) \stackrel{\text { tr }}{\longrightarrow} H^{i+1}(G) \stackrel{\cup v}{\longrightarrow} \cdots
$$

Proof. (Compare [D-M, Appendix 1]). The fibration $\mathbf{Z} / 2 \rightarrow B_{H} \rightarrow B_{G}$ is the sphere fibration of a line bundle. Consequently $M C(p)=B_{G} / B_{H}$ is the Thom space of the line bundle with first Stiefel-Whitney class $v$. Now apply the Gysin sequence. Transversality identifies

$$
B_{G} / B_{H} \rightarrow \Sigma B_{H}
$$

with the transfer. This can also be proved algebraically using the short sequence of $G$-modules

$$
0 \rightarrow \mathrm{F}_{2} \rightarrow \mathrm{F}_{2}[G / H] \rightarrow \mathrm{F}_{2} \rightarrow 0 .
$$

We apply this lemma to the situation $A_{n} \subseteq \Sigma_{n}$, with $\left[\Sigma_{n}: A_{n}\right]=2$. We know $H^{1}\left(\Sigma_{n}\right) \cong F_{2}$, generated by the symmetric class $\sigma_{1}$; hence 
Theorem 4.2. There is a short exact sequence of $H^{*}\left(\Sigma_{n}\right)$-modules:

$$
0 \longrightarrow H^{*}\left(\Sigma_{n}\right) /\left(\sigma_{1}\right) \stackrel{\text { res }}{\longrightarrow} H^{*}\left(A_{n}\right) \stackrel{\mathrm{tr}}{\longrightarrow} \mathrm{Ann}\left(\sigma_{1}\right) \longrightarrow 0 .
$$

From the considerations in Sect. 1, it is not hard to see that the ideal $J_{n}=\operatorname{Ann}\left(\sigma_{1}\right)$ is generated by "pure" Dickson classes. These can be described as follows: let $n=2^{j_{1}}+\cdots+2^{j_{r}}$, where $1 \leqq j_{1} \leqq \cdots \leqq j_{r}$ (the case $j_{1}=0$ can be reduced to this situation); the pure Dickson classes are those of the form $\left(Q_{I_{1}} * \cdots * Q_{I_{r}}\right)^{*}$, where $2 \leqq$ length $\left(I_{k}\right)=j_{k}$ for all $k=1, \ldots, r$ and each $I_{k}=(1, \ldots, 1)$.

An immediate consequence of this is the following

Corollary 4.3. If $n$ is congruent to 2 or $3 \bmod 4$, then the restriction map $H^{*}\left(\Sigma_{n}\right) \rightarrow H^{*}\left(A_{n}\right)$ is onto, and

$$
H^{*}\left(A_{n}\right) \cong H^{*}\left(\Sigma_{n}\right) /\left(\sigma_{1}\right) .
$$

We remark that the results in Sect. 1 together with the preceding discussion can be used to completely determine the additive structure of $H^{*}\left(A_{n}\right)$, any $n$. The ring structure is of course much harder, but we can recover the rings $H^{*}\left(A_{6}\right)$, $H^{*}\left(A_{8}\right), H^{*}\left(A_{10}\right)$ and $H^{*}\left(A_{12}\right)$ in what follows.

From 3.1 and 4.3 we have:

\section{Corollary 4.4 .}

$$
H^{*}\left(A_{6}\right) \cong P\left[\sigma_{2}, \sigma_{3}, c_{3}\right] /\left\langle c_{3} \sigma_{3}=0\right\rangle
$$

with

$$
S q^{1} \sigma_{2}=\sigma_{3}+c_{3}, S q^{1} c_{3}=0, S q^{2} c_{3}=\sigma_{2} c_{3}, S q^{1} \sigma_{3}=0, S q^{2} \sigma_{3}=\sigma_{3} \sigma_{2} .
$$

Proof. By 3.1, Ann $\left(\sigma_{1}\right)=0$, hence

$$
H^{*}\left(A_{6}\right) \cong H^{*}\left(\Sigma_{6}\right) /\left(\sigma_{1}\right)
$$

Applying 4.2 to $A_{8}$, we obtain the following

\section{Corollary 4.5.}

$$
H^{*}\left(A_{8}\right) \cong P\left[\sigma_{2}, c_{3}, \sigma_{3}, \sigma_{4}, d_{6}, e_{6}, d_{7}, e_{7}\right]\left(x_{5}\right) /\langle R\rangle
$$

where $\operatorname{deg} \sigma_{i}=i, \operatorname{deg} c_{3}=3, \operatorname{deg} d_{i}=i, \operatorname{deg} e_{i}=i, \operatorname{deg} x_{i}=5$, and $R$ is following set of relations:

$$
\begin{aligned}
& d_{6} \sigma_{3}=0, \quad d_{6} d_{7}+d_{6} e_{7}+e_{6} e_{7}=0, \quad d_{6}^{2}+d_{6} e_{6}+e_{6}^{2}=0, \\
& d_{7} \sigma_{2}=d_{7} \sigma_{3}=d_{7} c_{3}=d_{7} x_{5}=0, \quad d_{6} d_{7}+d_{7} e_{6}+e_{6} e_{7}=0, \quad d_{7}^{2}+d_{7} e_{7}+e_{7}^{2}=0, \\
& e_{6} \sigma_{3}=0, \\
& e_{7} \sigma_{2}=e_{7} \sigma_{3}=e_{7} c_{3}=e_{7} x_{5}=0, \\
& x_{5} \sigma_{3}=0, \quad c_{3} \sigma_{3}=0, \\
& x_{5}^{2}+x_{5} \sigma_{2} c_{3}+\left(d_{6}+e_{6}\right) \sigma_{2}^{2}+\sigma_{4} c_{3}^{2}=0 .
\end{aligned}
$$

The action of the Steenrod algebra on the generators different from $e_{6}, e_{7}$ can be read from 3.2 using the restriction map; for $e_{6}, e_{7}$ the action is identical to that on $d_{6}$ and $d_{7}$ substituting $e_{6}, e_{7}$ for $d_{6}, d_{7}$ throughout. 
Proof. Using 3.2 and 4.2, we have a short exact sequence

$$
0 \longrightarrow H^{*}\left(\Sigma_{8}\right) /\left(\sigma_{1}\right) \stackrel{\text { res }}{\longrightarrow} H^{*}\left(A_{8}\right) \stackrel{\text { r }}{\longrightarrow}\left(d_{6}, d_{7}\right) \rightarrow 0 .
$$

Here $\left(d_{6}, d_{7}\right)$ is the ideal generated by $d_{6}$ and $d_{7}$ in $H^{*}\left(\Sigma_{8}\right)$, and res is an isomorphism until degree 6 , where a new class $e_{6} \in H^{6}\left(A_{8}\right)$; similarly there is a new class $e_{7} \in H^{7}\left(A_{8}\right)$, and we have $\operatorname{tr}\left(e_{6}\right)=d_{6}, \operatorname{tr}\left(e_{7}\right)=d_{7}$. Note that $\operatorname{tr}(x y)=\operatorname{tr}(x) \cdot y$ for $y \in H^{*}\left(\Sigma_{8}\right) /\left(\sigma_{1}\right)$, hence we need only adjoin $e_{6}, e_{7}$ and their products with elements in im res to obtain $H^{*}\left(A_{8}\right)$. It only remains to determine the multiplicative relations involving these two classes.

Let $N_{\Sigma_{8}}\left(V_{3}\right), N_{A_{8}}\left(V_{3}\right)$ be the normalizers of $V_{3}$ in $\Sigma_{8}$ and $A_{8}$ respectively. Then $\left[\Sigma_{8}: N_{\Sigma_{8}}\left(V_{3}\right)\right]=2\left[A_{8}: N_{A_{8}}\left(V_{3}\right)\right]$ as there are twice as many conjugates of $V_{3}$ obtained by using elements in $\Sigma_{8}$ as those obtained by using only elements in $A_{8}$. Hence there exists $1 \neq \bar{g} \in \Sigma_{8} / A_{8}$ such that $V_{3}$ and $g V_{3} g^{-1}$ are not conjugate in $A_{8}$. The classes $e_{6}, e_{7}$ will be detected on $g V g^{-1}$. For this note that $N_{A_{8}}\left(V_{3}\right)=$ $N_{\Sigma_{8}}\left(V_{3}\right)=\operatorname{Aff}_{3}(\mathrm{Z} / 2)$; similarly $N_{A_{8}}\left(g V_{3} g^{-1}\right)=N_{\Sigma_{8}}\left(V_{3}\right)$. We have a diagram of restriction maps

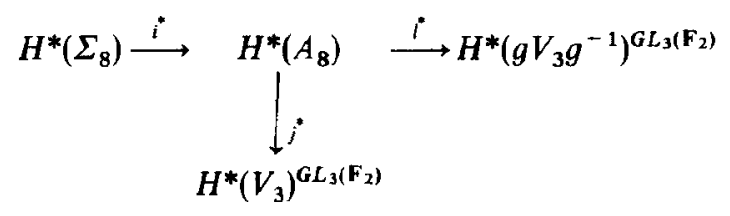

The maps $j^{*}$ and $l^{*}$ are onto: as noted before their images will be subpolynomial algebras generated by the Dickson invariants $D_{4}, D_{6}, D_{7}$ and $E_{4}, E_{6}, E_{7}$ respectively. The classes $D_{4}, E_{4}$ are identified with $\sigma_{4} \in H^{4}\left(A_{8}\right)$ and we obtain $d_{6}, e_{6}, d_{7}, e_{7} \in H^{*}\left(A_{8}\right)$ with $j^{*}\left(d_{6}\right)=D_{6}, j^{*}\left(d_{7}\right)=D_{7}, l^{*}\left(e_{6}\right)=E_{6}, l^{*}\left(e_{7}\right)=E_{7}$.

The element $\bar{g} \in \Sigma_{8} / A_{8}$ induces an involution on $H^{*}\left(A_{8}\right)$ with $\bar{g}^{*} d_{i}=e_{i}, i=6,7$; also note that res $d_{i}=d_{i}+e_{i}$. Now $\operatorname{tr}\left(\left(e_{i}+d_{i}\right) e_{i}\right)=\operatorname{tr}\left(\left(\operatorname{res} d_{i}\right) e_{i}\right)=d_{i} \cdot \operatorname{tr} e_{i}=d_{i}^{2}$. Therefore

$$
\operatorname{tr}\left(e_{i}^{2}+\left(e_{i}+d_{i}\right) e_{i}\right)=0 \text { and } e_{i} d_{i} \text { eim res. }
$$

Using the restriction to $V_{3}$ and $g V_{3} g^{-1}$, it follows that

$$
e_{i} d_{i}=\operatorname{res} d_{i}^{2}
$$

yielding the relation

$$
e_{i}^{2}+e_{i} d_{i}+d_{i}^{2}=0 .
$$

The same procedure yields the relations

$$
\begin{aligned}
& e_{6} e_{7}+d_{6} e_{7}+d_{6} d_{7}=0 \\
& e_{6} e_{7}+d_{7} e_{6}+d_{6} d_{7}=0 .
\end{aligned}
$$

The other relations, involving $e_{6}, e_{7}$ and the other generators, follow from the corresponding relations for $d_{6}, d_{7}$. The remaining relations follow from applying res to relations in $H^{*}\left(\Sigma_{8}\right)$. 
The explicit calculation of $H^{*}\left(A_{8}\right)$ is particularly interesting in light of the fact that $A_{8} \cong G L_{4}\left(\mathbf{F}_{2}\right)$. This isomorphism, due to Jordan and found in Dickson [D], can be described by the following correspondence of generators:

$$
\begin{array}{ll}
(23)(12) \mapsto\left(\begin{array}{llll}
1 & 1 & 1 & 1 \\
0 & 0 & 0 & 1 \\
1 & 1 & 0 & 0 \\
0 & 1 & 0 & 1
\end{array}\right), & (34)(12) \mapsto\left(\begin{array}{llll}
0 & 1 & 0 & 1 \\
0 & 0 & 1 & 0 \\
0 & 1 & 0 & 0 \\
1 & 0 & 1 & 0
\end{array}\right) \\
(45)(12) \mapsto\left(\begin{array}{llll}
0 & 1 & 1 & 1 \\
0 & 1 & 0 & 1 \\
1 & 1 & 0 & 0 \\
0 & 0 & 0 & 1
\end{array}\right), & (56)(12) \mapsto\left(\begin{array}{llll}
1 & 0 & 1 & 0 \\
0 & 1 & 0 & 0 \\
0 & 0 & 1 & 0 \\
0 & 1 & 0 & 1
\end{array}\right) \\
(67)(12) \mapsto\left(\begin{array}{llll}
0 & 0 & 1 & 0 \\
0 & 1 & 0 & 1 \\
1 & 0 & 0 & 0 \\
0 & 0 & 0 & 1
\end{array}\right), & (78)(12) \mapsto\left(\begin{array}{llll}
0 & 1 & 1 & 1 \\
0 & 0 & 1 & 0 \\
0 & 1 & 0 & 0 \\
1 & 1 & 1 & 0
\end{array}\right) .
\end{array}
$$

A calculation for $H^{*}\left(G L_{4}\left(\mathbf{F}_{2}\right)\right)$ was first attempted in [T-Y]; there was an error which was corrected later. However, at best their approach led to a listing of elements without giving any insight as to their significance or explicit multiplicative relations.

Corollary 4.6. The Poincaré series for $H^{*}\left(G L_{4}\left(\dot{\mathrm{F}}_{2}\right)\right)$ is

$$
P(t)=\frac{t^{15}-t^{14}+t^{13}-t^{12}+t^{11}-t^{9}+t^{8}+t^{7}+2 t^{6}+2 t^{4}-t^{3}+2 t^{2}-t+1}{\left(t^{7}-1\right)\left(t^{6}-1\right)\left(t^{3}-1\right)(t-1)\left(t^{2}+1\right)} .
$$

Using an analogous approach, we obtain

\section{Corollary 4.7.}

$$
H^{*}\left(A_{10}\right) \cong P\left[\sigma_{2}, \sigma_{3}, \sigma_{4}, \sigma_{5}, c_{3}, x_{5}, d_{6}\right] /\langle R\rangle
$$

where $R$ is the set of relations

$$
\begin{aligned}
& \sigma_{5} d_{7}, \sigma_{3} d_{7}, c_{3} d_{7}, x_{5} d_{7}, \sigma_{3} d_{6}, \sigma_{5} d_{6} \\
& x_{5}^{2}+\sigma_{2} c_{3} x_{5}+c_{3}^{2} \sigma_{4}+\sigma_{2}^{2} d_{6} \\
& \sigma_{5} x_{5}+c_{3} \sigma_{3} \sigma_{4}, \sigma_{3} x_{5}+c_{3}\left(\sigma_{5}+\sigma_{2} \sigma_{3}\right) .
\end{aligned}
$$

As for $A_{6}$, the action of the Steenrod algebra on the generators follows directly from the description of $H^{*}\left(\Sigma_{10}\right)$.

\section{Corollary 4.8.}

$$
H^{*}\left(A_{12}\right) \cong P\left[\sigma_{2}, \sigma_{3}, c_{3}, \sigma_{4}, \sigma_{5}, \sigma_{6}, d_{6}, d_{7}, d_{9}, e_{9}, e_{10}\right]\left(x_{5}, x_{7}, x_{8}\right) /\langle R\rangle
$$

where $\operatorname{deg} \sigma_{i}=i, \operatorname{deg} c_{3}=3, \operatorname{deg} d_{i}=i, \operatorname{deg} x_{i}=i, \operatorname{deg} e_{i}=i$ and $R$ is the set of 
relations

$$
\begin{aligned}
& x_{7}^{2}+\sigma_{4} x_{5}^{2}+\sigma_{2} \sigma_{4} x_{8}+\left(\sigma_{6} c_{3}+\sigma_{2} \sigma_{4} c_{3}\right) x_{5}+\sigma_{4}^{2}+c_{3}^{2}+\sigma_{2}^{2} \sigma_{4} d_{6}+\sigma_{6} \sigma_{2} d_{6}, \\
& x_{8}^{2}+d_{6} x_{5}^{2}+c_{3} d_{6} x_{7}+\left(\left[e_{9}+d_{9}\right] \sigma_{2}+c_{3} d_{6} \sigma_{2}\right) x_{5}+d_{6}^{2} \sigma_{2}^{2}+c_{3}^{2} \sigma_{4} d_{6}+\left[e_{9}+d_{9}\right] c_{3} \sigma_{2}, \\
& x_{5} x_{7}+\sigma_{2} x_{5}^{2}+\left[\sigma_{2}^{2}+\sigma_{4}\right] x_{8}+\sigma_{2}^{2} c_{3} x_{5}+\sigma_{2}^{3} d_{6}+\sigma_{2} d_{6} \sigma_{4}+\sigma_{6} c_{3}^{2}+\sigma_{6} d_{6}+\sigma_{2} \sigma_{4} c_{3}^{2}, \\
& x_{5} x_{8}+c_{3} x_{5}^{2}+\left[c_{3}^{2}+d_{6}\right] x_{7}+c_{3}^{2} \sigma_{2} x_{5}+c_{3}^{3} \sigma_{4}+c_{3} \sigma_{4} d_{6}+\left[e_{9}+d_{9}\right] \sigma_{2}^{2}+\left[e_{9}+d_{9}\right] \sigma_{4} \\
& \quad+c_{3} d_{6} \sigma_{2}^{2}, \\
& x_{5}^{3}+c_{3} \sigma_{2} x_{5}^{2}+x_{7} x_{8}+c_{3} \sigma_{2}^{2} x_{8}+\sigma_{2} c_{3}^{2} x_{7}+\left(\sigma_{2}^{2} d_{6}+\sigma_{4} c_{3}^{2}\right) x_{5}+\sigma_{2}^{2}\left[e_{9}+d_{9}\right]+c_{3}^{3} \sigma_{6} \\
& \quad+\sigma_{6}\left[e_{9}+d_{9}\right], \\
& d_{9} \sigma_{3}, \quad d_{9} \sigma_{5}, \quad e_{9} \sigma_{3}, \quad e_{9} \sigma_{5}, \\
& d_{7} \sigma_{3}, \quad d_{7} x_{5}, \quad e_{10} \sigma_{4}+d_{7}\left(x_{7}+\sigma_{4} c_{3}\right), \quad d_{7} \sigma_{5}, \quad d_{7}\left(\sigma_{6}+\sigma_{4} \sigma_{2}\right), \quad d_{7}\left(x_{8}+d_{6} \sigma_{2}\right), \\
& d_{7}\left(e_{9}+d_{9}\right)+d_{6}\left(d_{7} c_{3}+e_{10}\right), \\
& x_{5} \sigma_{3}+c_{3}\left(\sigma_{2} \sigma_{3}+\sigma_{5}\right), \quad x_{7} \sigma_{3}+x_{5} \sigma_{5}+c_{3} \sigma_{3} \sigma_{4}, \quad x_{7} \sigma_{5}+c_{3} \sigma_{3} \sigma_{6}, \\
& d_{6} \sigma_{3}, \quad x_{8} \sigma_{3}+d_{6} \sigma_{5}, \quad x_{8} \sigma_{5}, \\
& d_{9}^{2}+d_{9} e_{9}+e_{9}^{2}, \quad\left(d_{7} c_{3}\right)^{2}+d_{7} c_{3} e_{10}+e_{10}^{2}, \\
& d_{9} d_{7} c_{3}+d_{9} e_{10}+e_{9} e_{10}, \quad d_{9} d_{7} c_{3}+d_{7} c_{3} e_{9}+e_{9} e_{10} .
\end{aligned}
$$

The action on the Steenrod algebra on generators different from $e_{9}, e_{10}$ can be obtained from 3.4 using the restriction map; for $e_{9}, e_{10}$ the action is identical to that on $d_{9}$ and $d_{7} c_{3}$ doing the appropriate substitutions.

\section{References}

[AMM] Adem, A., Maginnis, J., Milgram, R.J.: The geometry and cohomology of the Mathieu group $M_{12}$. J. Algebra, to appear

[B-P] Barratt, M.G., Priddy, S.: On the homology of non-connected monoids and their associated groups. Commut. Math. Helv. 47, 1-14 (1972)

[D] Dickson, L.E.: The alternating group on eight letters and the quaternary linear congruence group modulo two. Math. Ann. 54, 564-569 (1901)

[D-L] Dyer, E., Lashof, R.: Homology of iterated loop spaces. Am. J. Math. 84, 35-88 (1962)

[D-M] Davis, J., Milgram, R.J.: Semicharacteristics, bordism and free group actions. Trans. Am. Math. Soc. 312, 55-84 (1989)

[E] Evens, L.: The cohomology ring of a finite group. Trans. Am. Math. Soc. 101, 224-239 (1961)

[H] Hung, N.H.V.: The modulo 2 cohomology algebras of the symmetric groups. Jap. J. Math. 13, 169-208 (1987)

[M1] Milgram, R.J.: The mod 2 spherical characteristic classes. Ann. Math. 92, 238-261 (1970)

[M2] Milgram, R.J.: A remark on the Kirby-Siebenmann class. Preprint 1987

[M-M] Madsen, I., Milgram, R.J.: The classifying spaces for surgery and cobordism of manifolds. Ann. Math. Stud. 92 (1979)

[N] Nakaoka, M.: Homology of the infinite symmetric group. Ann. Math. 73, 229-257 (1961)

[Q] Quillen, D.: On the completion of a simplicial monoid. preprint MIT (1971)

[Q-V] Quillen, D., Venkov, B.B.: Cohomology of finite groups and elementary abelian subgroups. Topology 11, 317-318 (1971)

[T-Y] Tezuka, M., Yagita, N.: The cohomology of subgroups of $G L_{n}\left(F_{q}\right)$. Contmp. Math. 19, 379-396 (1983) 